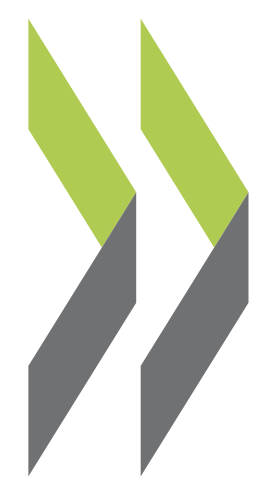

OECD Science, Technology and Industry Working Papers $2013 / 05$

\title{
A Cross-Country Characterisation of the Patenting Behaviour of Firms based on Matched Firm and Patent Data
}

Mariagrazia Squicciarini, Hélène Dernis 
Organisation de Coopération et de Développement Économiques

Organisation for Economic Co-operation and Development

10-Sep-2013

DIRECTORATE FOR SCIENCE, TECHNOLOGY AND INDUSTRY

English - Or. English

\section{A CROSS-COUNTRY CHARACTERISATION OF THE PATENTING BEHAVIOUR OF FIRMS BASED ON MATCHED FIRM AND PATENT DATA}

STI Working Paper 2013/5

By Mariagrazia Squicciarini and Hélène Dernis (OECD)

Complete document available on OLIS in its original format

This document and any map included herein are without prejudice to the status of or sovereignty over any territory, to the delimitation of international frontiers and boundaries and to the name of any territory, city or area. 


\section{STI WORKING PAPER SERIES}

The Working Paper series of the OECD Directorate for Science, Technology and Industry is designed to make available to a wider readership selected studies prepared by staff in the Directorate or by outside consultants working on OECD projects. The papers included in the series cover a broad range of issues, of both a technical and policy-analytical nature, in the areas of work of the DSTI. The Working Papers are generally available only in their original language - English or French - with a summary in the other.

Comments on the papers are invited, and should be sent to the Directorate for Science, Technology and Industry, OECD, 2 rue André-Pascal, 75775 Paris Cedex 16, France.

The opinions expressed in these papers are the sole responsibility of the author(s) and do not necessarily reflect those of the OECD or of the governments of its member countries.

www.oecd.org/sti/working-papers

OECD/OCDE, 2013

Applications for permission to reproduce or translate all or part of this material should be made to: OECD Publications, 2 rue André-Pascal, 75775 Paris, Cedex 16, France; e-mail: rights@oecd.org 
TABLE OF CONTENTS

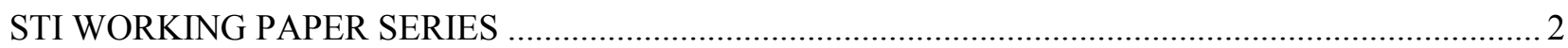

A CROSS-COUNTRY CHARACTERISATION OF THE PATENTING BEHAVIOUR OF FIRMS BASED ON MATCHED FIRM AND PATENT DATA ….................................................. 4

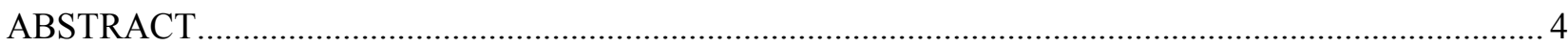

CATÉGORISER LE COMPORTEMENT DES ENTREPRISES DÉPOSANT DES BREVETS :

UNE ÉTUDE FONDÉE SUR L'APPARIEMENT ENTRE LES DONNÉES D'ENTREPRISES ET DE

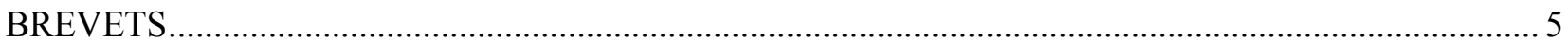

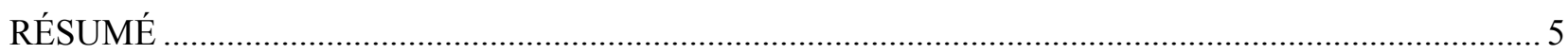

A CROSS-COUNTRY CHARACTERISATION OF THE PATENTING BEHAVIOUR OF

FIRMS BASED ON MATCHED FIRM AND PATENT DATA …............................................................ 6

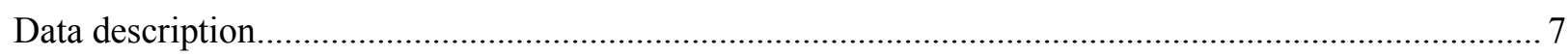

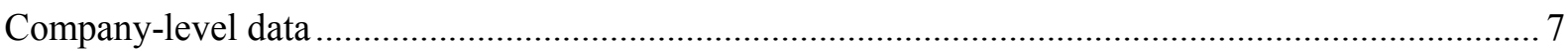

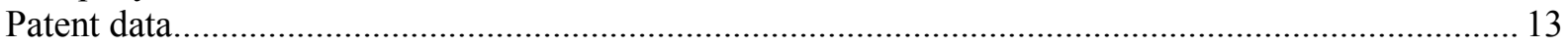

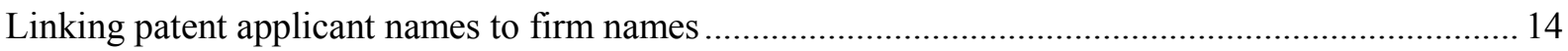

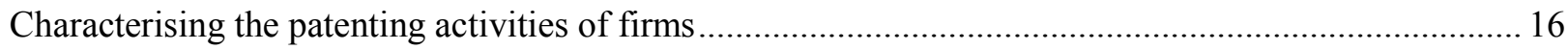

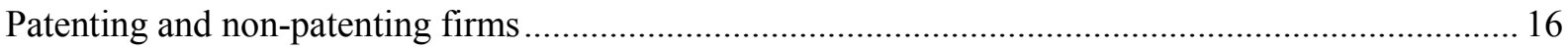

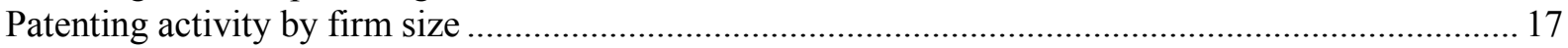

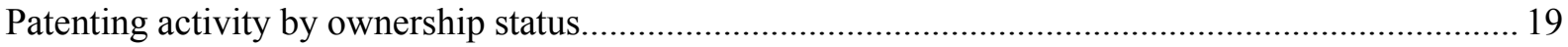

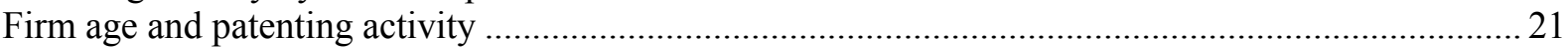

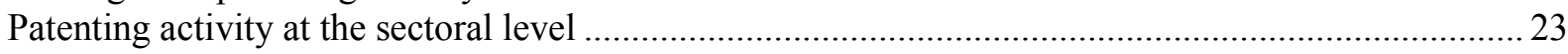

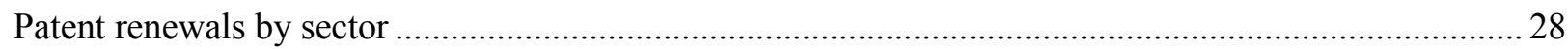

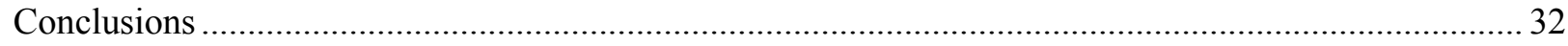

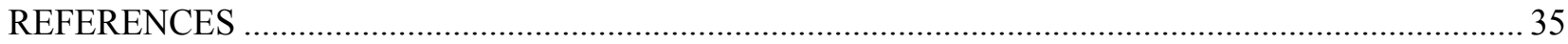


DSTI/DOC(2013)5

\title{
A CROSS-COUNTRY CHARACTERISATION OF THE PATENTING BEHAVIOUR OF FIRMS BASED ON MATCHED FIRM AND PATENT DATA
}

\author{
Mariagrazia Squicciarini, Hélène Dernis
}

\begin{abstract}
This work proposes a characterisation of the patenting behaviours of firms. It relies on patent data linked to firm data from a commercial dataset, regards firms of 20 or more employees located in 15 countries, and refers to the period 1999-2010. The way in which patent assignees' names are linked to firm names is explained, and the coverage and representativeness of the firm database used is discussed using information from structural business statistics. The profile of patenting and non-patenting firms is delineated on the basis of characteristics such as firm size, ownership, firm age and industry, and of combinations thereof. Statistics related to the sector-specific patterns of patent renewals are also shown. Evidence suggests that only $0.1 \%-9.3 \%$ of firms apply for patents, with marked differences that exist between industries and across countries. Small and medium enterprises account for the majority of patenting firms, while big firms of more than 250 employees account for the majority of patent applications and of patent families. First-time patenting is typically observed to occur during the first ten years of a firm's life. Patent renewal patterns appear sector specific, although a significant proportion of patents (14\%-26\%) gets renewed up to 16-20 years in all technology fields.
\end{abstract}


DSTI/DOC(2013)5

\title{
CATÉGORISER LE COMPORTEMENT DES ENTREPRISES DÉPOSANT DES BREVETS : UNE ÉTUDE FONDÉE SUR L'APPARIEMENT ENTRE LES DONNÉES D'ENTREPRISES ET DE BREVETS
}

\author{
Mariagrazia Squicciarini, Hélène Dernis
}

\author{
RÉSUMÉ
}

Ce travail vise à catégoriser le comportement des entreprises en matière de dépôt de brevets. Il repose sur la mise en relation de données de brevets avec les données d'entreprises extraites d'une source commerciale, permettant ainsi d'observer les entreprises de plus de 20 salariés dans 15 pays sur la période 1999-2010. Les techniques d'appariement entre les noms des détenteurs de brevets et les noms d'entreprises y sont détaillées. La couverture et la représentativité des données d'entreprises utilisées sont confrontées aux informations fournies par les statistiques structurelles d'entreprises. Le profil des entreprises déposant ou non des brevets est retracé selon des caractéristiques de taille, d'appartenance à un groupe, d'âge et/ou de secteur industriel. Des statistiques sur le maintien en vigueur des brevets sont également présentées par secteur économique. Les chiffres suggèrent que seuls $0.1 \%$ à $9.3 \%$ des entreprises déposent des brevets, avec des disparités marquées entre secteurs industriels et entre pays. Si les petites et moyennes entreprises représentent la majorité des entreprises déposant des brevets, les grandes entreprises de plus de 250 salariés détiennent la plupart des demandes de brevets et des familles de brevets. Le premier dépôt de brevet par une entreprise se produit généralement au cours des dix premières années d'activité de l'entreprise. Le maintien en vigueur des brevets apparait lié au secteur industriel de l'entreprise, bien qu'une proportion significative de brevets (entre $14 \%$ et $26 \%$ ) soit renouvelée jusque 16-20 ans, quel que soit le domaine technologique de l'invention. 


\section{A CROSS-COUNTRY CHARACTERISATION OF THE PATENTING BEHAVIOUR OF FIRMS BASED ON MATCHED FIRM AND PATENT DATA}

Patents have been shown to be positively associated with the survival of firms (Wagner and Cockburn, 2010) and to have an economically and statistically significant impact on firm-level productivity and market value (e.g. Bloom and Van Reenen, 2002). The decision to patent also appears to correlate with firms' subsequent growth in high- and medium-tech start-ups (Helmers and Rogers, 2011). ${ }^{1}$ Patents have further been found to increase the likelihood of obtaining venture capital and to secure liquidity through initial public offerings, especially in earlier financing rounds (see Hall and Harhoff, 2012, for a survey on the economics of patents).

The vast literature dealing with the use of patents by firms e.g. to appropriate the returns of investment in research and development (R\&D), deter entry, or secure financing, and with the way patenting activities relate to firm performance, typically focuses on one or few technological domains, industries, or countries at a time, often due to data availability constraints. The results of such studies, although providing very valuable insights, remain somewhat narrow in scope and cannot be generalised to other countries or industries. This impinges on the ability to uncover common patterns and possible determinants, benchmark performances across countries and over time, investigate the way countryspecific framework conditions shape patenting and firm dynamics and, ultimately, provide evidence in support of policy making of today's knowledge-based open economies.

This work aims to contribute to address such shortcomings. It proposes an exploratory characterisation of firms' patenting behaviours across fifteen countries over the period 1999-2010. The stylised facts proposed are meant to serve as a basis for broader policy relevant analyses, and as a starting point for a more informed discussion on the role of country-specific framework conditions in explaining the observed differences in firm behaviours and outcomes.

The descriptive nature of this analysis is dictated by the desire to offer some initial cross-country evidence on how firms of different size and age innovate and perform, while leaving selectivity, endogeneity and simultaneity concerns aside. No causal interpretation of the patterns observed can be inferred from the commercial firm database this work relies upon: in the impossibility to access and pool country-specific business register data, commercial data sources - namely ORBIS (C) data - are used, as a second best option. These commercial data however do not cover the entire universe of firms, and their representativeness varies across age and size classes, and across countries and over time.

To try and minimise the selectivity bias that ORBISC coverage might determine, this study is confined to countries (namely: Austria, Belgium, Canada, Finland, France, Germany, Ireland, Italy, Japan, Netherlands, Norway, Spain, Sweden, Switzerland, the United States) with highest coverage and representativeness throughout the period considered. Moreover, before presenting the patent-related analysis, a number of statistics are shown, which describe some of the main characteristics and shortcomings of ORBIS(C) as a source of firm-level data. The results of this simple exercise underline the limits inherent in using commercial databases to carry out robust micro-level analysis, and argue in favour

1. They estimate that patenting start-ups enjoy $8 \%-27 \%$ per annum higher asset growth than non-patentees. 
of using business register data instead, as they supply high-quality information in most cases related to the entire universe of firm in a country, at each point in time considered.

The cross-country characterisation of the patenting behaviour and performance of firms proposed in the present document has therefore to be considered as a first step towards a better understanding of the innovative behaviour of enterprises, while bearing in mind that its generality and robustness might not be fully assured. While in the impossibility to thoroughly assess ORBIS $\odot$ coverage and possible selection issues, no reasons a priori exist to believe that systematic sector-, size- and age-specific biases should exist. An implicit assumption about the existence of normally distributed random errors is hence made, and statistics are believed not to be off the mark.

Small representativeness concerns conversely exist with respect to the patent data used for the analysis: the OECD Patent database covers bibliographic records on patent applications filed in a large number of Intellectual Property (IP) Offices worldwide. It is mainly based on the PATSTAT database, which provides a comprehensive coverage of patents filed at the most important patent offices worldwide, including the European Patent Office (EPO), the US Patent and Trademark Office (USPTO), and patents filed under the Patent Co-Operation Treaty (PCT). Finally, in the absence of firms' identifiers in the patent documents, patent assignees' names have been linked to firm names by means of using a purposely built algorithm aimed at maximising matches, while minimising false positives and false negatives.

The remainder of this paper first describes the data sources used for the analysis, discusses some representativeness issues and illustrates how firm and patent data have been linked. It then delineates the profile of patenting and non-patenting firms. A characterisation of the patenting activity of firms by firm size, ownership status, firm age and industry is also proposed. Finally, statistics related to the sectorspecific patterns of patent renewals are shown, followed by some final remarks.

\section{Data description}

\section{Company-level data}

ORBIS $\odot$ is a commercial database produced by Bureau van Dijk Electronic Publishing (BvD) containing demographic, structural and financial information of firms located worldwide, mainly gathered from national public business registers. Depending on the version considered, ORBIS $\subset$ contains information relating to 50 million or more firms, as coverage seemingly increased over time, both on the intensive (i.e. adding more firms from a country already included in previous versions of the dataset) and the extensive margin (i.e. adding data related to firms belonging to countries not covered in previous versions).

The data contained in ORBISC have been organised at the OECD according to the type of information provided: demographic data, including e.g. name, location, activity sector, incorporation date; financial information obtained from balance sheets for the period 1999-2011; profit and loss accounts reported for given time periods; and information related to ownership and the consolidation structure of a group, if applicable. A company identifier, called BVDEP ID, allows linking the information contained in the different types of accounts, at both the consolidated and unconsolidated levels.

The ORBISC database exploited in the present analysis refers to a version made available to the OECD under licence in June 2011 (hereafter named OECD-ORBIS 2011 database). Our choice is motivated by the extended firm coverage of this version vis-à-vis earlier versions of the same database. Differences in the coverage within and across countries however exist, in terms of absolute number of firms covered (Table 1), and of availability of information gathered from the balance sheets (Table 2). 
As can be seen from Table 1, absolute coverage of firms providing financial information has generally increased over time, for all countries considered. The much smaller figures shown for the year 2010 may likely be due to factors like end-of-fiscal-year dates, which are known to vary across countries and within and across sectors; and data provision dates, i.e. the dates or periods in which Bureau van Dijk receives yearly records from its data suppliers. Factors like these lead to truncation in the distribution observed for the latest year available, i.e. 2010. This motivates the choice made in the present work to focus on the year 2009 when proposing a number of descriptive statistics and discussing representativeness issues.

Table 1. Number of firms providing financial information OECD-Orbis 2011, thousands

\begin{tabular}{lrrrrrrrrrrrr}
\hline & $\mathbf{1 9 9 9}$ & $\mathbf{2 0 0 0}$ & $\mathbf{2 0 0 1}$ & $\mathbf{2 0 0 2}$ & $\mathbf{2 0 0 3}$ & $\mathbf{2 0 0 4}$ & $\mathbf{2 0 0 5}$ & $\mathbf{2 0 0 6}$ & $\mathbf{2 0 0 7}$ & $\mathbf{2 0 0 8}$ & $\mathbf{2 0 0 9}$ & $\mathbf{2 0 1 0}$ \\
\hline Austria & 15.0 & 17.7 & 26.8 & 28.4 & 43.8 & 93.2 & 120.3 & 134.9 & 138.8 & 156.9 & 135.4 & 83.1 \\
Belgium & 83.0 & 231.4 & 271.8 & 283.3 & 293.3 & 303.5 & 312.7 & 330.4 & 343.5 & 356.6 & 365.1 & 79.1 \\
Canada & 2.4 & 3.6 & 7.2 & 14.0 & 76.3 & 139.4 & 717.9 & 838.2 & 836.0 & 858.2 & 867.5 & 675.0 \\
Finland & 25.9 & 51.4 & 69.8 & 78.9 & 90.0 & 90.4 & 91.5 & 97.4 & 128.4 & 149.6 & 147.7 & 51.1 \\
France & 128.5 & 151.8 & 719.5 & 778.4 & 803.4 & 843.0 & 881.0 & 984.6 & 1038.7 & 1070.4 & 1742.6 & 261.0 \\
Germany & 63.9 & 78.5 & 107.5 & 134.8 & 197.8 & 282.6 & 738.6 & 1049.5 & 1057.4 & 1086.3 & 1041.3 & 256.6 \\
Ireland & 13.0 & 44.7 & 91.3 & 97.7 & 103.3 & 108.2 & 111.8 & 114.3 & 123.7 & 124.0 & 88.1 & 2.0 \\
Italy & 64.6 & 139.7 & 166.1 & 259.9 & 241.9 & 528.2 & 551.0 & 586.2 & 906.7 & 925.1 & 915.1 & 82.7 \\
Japan & 3.3 & 41.0 & 90.4 & 112.9 & 156.0 & 197.0 & 258.7 & 339.4 & 685.3 & 1056.8 & 1036.0 & 538.2 \\
Netherlands & 126.2 & 183.0 & 203.9 & 267.0 & 284.1 & 303.2 & 320.7 & 344.4 & 349.0 & 351.7 & 403.2 & 45.9 \\
Norway & 54.1 & 132.1 & 142.6 & 141.6 & 141.9 & 144.8 & 160.7 & 211.7 & 224.8 & 236.2 & 238.3 & 36.1 \\
Spain & 234.7 & 432.1 & 547.4 & 666.0 & 713.5 & 719.8 & 744.5 & 790.6 & 708.0 & 774.4 & 663.2 & 0.7 \\
Sweden & 49.1 & 121.8 & 207.5 & 221.1 & 234.1 & 244.5 & 252.4 & 262.9 & 281.4 & 310.2 & 784.6 & 405.3 \\
Switzerland & 2.3 & 4.0 & 4.7 & 21.6 & 4.7 & 27.1 & 26.9 & 28.9 & 175.2 & 205.9 & 200.9 & 13.3 \\
United States & 9.0 & 11.1 & 29.6 & 31.0 & 190.8 & 368.6 & 669.1 & 903.9 & 6091.1 & 7072.2 & 12766.9 & 9479.5 \\
\hline
\end{tabular}

Source: OECD, calculations based on OECD-ORBISC June 2011, October 2012.

Table 2 further highlights the wide differences existing in the extent to which employee and turnover related data are provided. On average, turnover and employee-related figures are provided to a similar extent (in $64 \%$ and $65 \%$ of cases, respectively), although these patterns are not consistent across countries. The share of firms for which employee data are reported ranges between $18 \%$ (Ireland) and $100 \%$ (United States and Switzerland), whereas turnover data are provided by a proportion of firms varying between 3\% (the Netherlands) and 100\% (France, Japan and Norway). The proportion of firms for which both employees and turnover data are provided varies between $2 \%$ and $98 \%$, with an average figure for the countries considered that corresponds to $44 \%$ of the firms in ORBISC .

Table 2. Number of firms reporting figures on employees or turnover in 2009 OECD-Orbis 2011, thousands

\begin{tabular}{l|rl|rr|rr|r}
\hline & \multicolumn{2}{|c|}{ Employees } & \multicolumn{2}{|c|}{ Turnover } & \multicolumn{2}{|c}{$\begin{array}{c}\text { Employees and } \\
\text { Turnover }\end{array}$} & All firms* \\
\hline Austria & 92.8 & $69 \%$ & 19.8 & $15 \%$ & 10.9 & $8 \%$ & 135.4 \\
Belgium & 138.9 & $38 \%$ & 75.8 & $21 \%$ & 37.6 & $10 \%$ & 365.1 \\
Canada & 862.2 & $99 \%$ & 822.0 & $95 \%$ & 817.9 & $94 \%$ & 867.5 \\
Finland & 46.0 & $31 \%$ & 140.4 & $95 \%$ & 45.8 & $31 \%$ & 147.7 \\
France & 1368.5 & $79 \%$ & 1742.5 & $100 \%$ & 1368.4 & $79 \%$ & 1742.6 \\
Germany & 257.0 & $25 \%$ & 383.4 & $37 \%$ & 107.3 & $10 \%$ & 1041.3 \\
Ireland & 15.8 & $18 \%$ & 11.2 & $13 \%$ & 2.9 & $3 \%$ & 88.1 \\
Italy & 308.7 & $34 \%$ & 907.8 & $99 \%$ & 308.7 & $34 \%$ & 915.1 \\
Japan & 221.1 & $21 \%$ & 1036.0 & $100 \%$ & 221.1 & $21 \%$ & 1036.0 \\
Netherlands & 311.5 & $77 \%$ & 12.5 & $3 \%$ & 8.4 & $2 \%$ & 403.2 \\
Norway & 234.4 & $98 \%$ & 238.2 & $100 \%$ & 234.4 & $98 \%$ & 238.3 \\
Spain & 508.7 & $77 \%$ & 605.5 & $91 \%$ & 503.1 & $76 \%$ & 663.2 \\
Sweden & 770.5 & $98 \%$ & 761.2 & $97 \%$ & 747.7 & $95 \%$ & 784.6 \\
Switzerland & 200.6 & $100 \%$ & 18.3 & $9 \%$ & 18.0 & $9 \%$ & 200.9 \\
United States & 12708.7 & $100 \%$ & 11968.9 & $94 \%$ & 11910.8 & $93 \%$ & 12766.9 \\
\hline
\end{tabular}

Note: * Data refer to all firms reporting at least one piece of financial information.

Source: OECD, calculations based on OECD-ORBIS@ June 2011, October 2012. 
A comparison of ORBISC with Structural Business Statistics (SBS) figures further highlights the heterogeneity of the proportion of firms included in ORBIS by size class. Table 3 a shows data related to Austria, Belgium, Finland, France, Germany and Ireland, whereas Table $3 \mathrm{~b}$ proposes the same statistics for Italy, the Netherlands, Spain, Norway, Sweden, and Switzerland. Firms are grouped into 5 size classes: micro firms featuring 0 to 9 employees; very small firms with 10-19 employees; small firms of 20-49 employees; medium firms with 50 to 249 employees; and big firms with 250 employees or more. For each size class considered, Tables $3 \mathrm{a}$ and Table $3 \mathrm{~b}$ show the sector-specific number of firms included in ORBISC and the corresponding share of firms included in the National SBS that these figures should correspond to. The last columns on the right hand size of Table $3 \mathrm{a}$ and Table $3 \mathrm{~b}$ show the same figures at the aggregate sectoral level, i.e. irrespective of firm size.

For those countries for which representativeness could be assessed, large differences in coverage emerge, as well some inconsistencies - notably coverage above $100 \%$. These may be due to a number of factors, including: the level of consolidation at which data are provided or presented in the commercial database; the ways in which statistical agencies classify firms and the possible differences that exist with respect to the classification criteria used in the public registers that ORBISC relies upon; the way in which sample attrition and (re)entry into the sample is dealt with in ORBISC), and the extent to which re-entering firms are identified and duplications avoided; possible different sector allocations, in the case of diversified enterprises; sector misallocation due to e.g. converting industry codes from a different statistical classification (NACE Rev 1.1 to NACE Rev. 2); public or private ownership, as SBS only accounts for privately owned firms, whereas ORBISC does not. Evidently, percentages can go easily above $100 \%$ if sector misallocation applies to companies in sectors featuring a small number of firms (e.g. in mining and quarrying), whereas this should not be the case in those sectors populated by a relatively higher number of firms (e.g. manufacturing).

For those firms for which employment related data are not available while related pieces of information are (the cost of employees), the imputed employee figures calculated by Ragoussis and Gonnard (2012) are used. In addition, in the case that employment related data appear missing for the focal year considered, i.e. 2009, but are available for any of the three preceding years or in 2010, the closest in time employee-related observation available is imputed.

The figures shown in Table $3 \mathrm{a}$ and Table $3 \mathrm{~b}$ call for additional investigation to understand the actual reasons leading ORBIS $\odot$ to exhibit such figures, and suggest that care should be used when interpreting the results of studies based on commercial data sources. The limited availability of additional information in ORBIS $\odot$ in fact hinders a full appreciation of the statistical properties of the sample at hand and the possibility to properly address selectivity issues. These shortcomings motivate the choice of the present analysis to focus on enterprises of 20 employees or more, given that coverage looks less heterogeneous across countries and sectors for these size classes. The final aim is to try and minimise the biases that representativeness issues may trigger, while maximising the scope and coverage of the study. 
Table 3a. Firms by size class and industry - comparing ORBIS $\odot$ and structural business statistics data, 2009

\begin{tabular}{|c|c|c|c|c|c|c|c|c|c|c|c|c|}
\hline \multirow[b]{2}{*}{ Nace, rev. 2} & \multicolumn{2}{|c|}{0 to 9} & \multicolumn{2}{|c|}{10 to 19} & \multicolumn{2}{|c|}{20 to 49} & \multicolumn{2}{|c|}{50 to 249} & \multicolumn{2}{|c|}{$250+$} & \multicolumn{2}{|c|}{ All firms* } \\
\hline & $\begin{array}{c}\text { Firms } \\
\text { in ORBIS }\end{array}$ & $\begin{array}{c}\% \\
\text { of SBS }\end{array}$ & $\begin{array}{c}\text { Firms } \\
\text { in ORBIS }\end{array}$ & $\begin{array}{c}\% \\
\text { of SBS }\end{array}$ & $\begin{array}{c}\text { Firms } \\
\text { in ORBIS }\end{array}$ & $\begin{array}{c}\% \\
\text { of SBS }\end{array}$ & $\begin{array}{c}\text { Firms } \\
\text { in ORBIS }\end{array}$ & \begin{tabular}{c|}
$\%$ \\
of SBS
\end{tabular} & $\begin{array}{c}\text { Firms } \\
\text { in ORBIS }\end{array}$ & \begin{tabular}{c|}
$\%$ \\
of SBS
\end{tabular} & $\begin{array}{c}\text { Firms } \\
\text { in ORBIS }\end{array}$ & $\begin{array}{c}\% \\
\text { of SBS }\end{array}$ \\
\hline \multicolumn{13}{|l|}{ Austria } \\
\hline B - Mining and Quarrying & 0.20 & $83 \%$ & 0.06 & $145 \%$ & 0.06 & $118 \%$ & 0.04 & $216 \%$ & 0.01 & $180 \%$ & 0.37 & $103 \%$ \\
\hline C - Manufacturing & 11.47 & $63 \%$ & 2.68 & $87 \%$ & 2.25 & $105 \%$ & 1.56 & $107 \%$ & 0.43 & $96 \%$ & 18.39 & $73 \%$ \\
\hline D - Electricity, Gas, Steam and Air Conditioning Supply & 0.63 & $44 \%$ & 0.04 & $116 \%$ & 0.03 & $90 \%$ & 0.03 & $68 \%$ & 0.02 & $71 \%$ & 0.74 & $47 \%$ \\
\hline E - Water supply; Sewerage, Waste Management (...) & 0.48 & $29 \%$ & 0.10 & $68 \%$ & 0.10 & $96 \%$ & 0.05 & $131 \%$ & 0.01 & $75 \%$ & 0.72 & $38 \%$ \\
\hline H - Transportation and Storage & 6.30 & $56 \%$ & 1.08 & $80 \%$ & 0.89 & $104 \%$ & 0.38 & $114 \%$ & 0.06 & $105 \%$ & 8.71 & $63 \%$ \\
\hline I- Accomodation and Food Service Activities & 13.01 & $33 \%$ & 2.10 & $63 \%$ & 1.34 & $97 \%$ & 0.43 & $100 \%$ & 0.04 & $157 \%$ & 16.92 & $38 \%$ \\
\hline $\mathrm{J}$ - Information and Communication & 7.34 & $51 \%$ & 0.53 & $97 \%$ & 0.37 & $102 \%$ & 0.18 & $108 \%$ & 0.03 & $123 \%$ & 8.46 & $55 \%$ \\
\hline L - Real Es tate Activities & 7.13 & $47 \%$ & 0.25 & $97 \%$ & 0.17 & $113 \%$ & 0.09 & $108 \%$ & 0.02 & $150 \%$ & 7.65 & $48 \%$ \\
\hline M-Professional, Scientific and Technical Activities & 25.60 & $49 \%$ & 1.48 & $67 \%$ & 0.86 & $97 \%$ & 0.32 & $116 \%$ & 0.05 & $186 \%$ & 28.32 & $51 \%$ \\
\hline $\mathrm{N}$ - Administrative and Support Service Activities & 54.78 & $549 \%$ & 1.25 & $145 \%$ & 0.81 & $128 \%$ & 0.49 & $113 \%$ & 0.11 & $111 \%$ & 57.44 & $478 \%$ \\
\hline \multicolumn{13}{|l|}{ Belgium } \\
\hline B - Mining and Quarrying & 0.15 & $60 \%$ & 0.03 & & 0.03 & $227 \%$ & 0.02 & $178 \%$ & 0.00 & & 0.22 & \\
\hline C - Manufacturing & 13.50 & $43 \%$ & 2.32 & $82 \%$ & 2.06 & $92 \%$ & 1.33 & & 0.31 & & 19.53 & $51 \%$ \\
\hline D - Electricity, Gas, Steam and Air Conditioning Supply & 0.09 & $49 \%$ & 0.01 & & 0.01 & $133 \%$ & 0.01 & & 0.01 & & 0.14 & \\
\hline E - Water supply; Sewerage, Waste Management (...) & 0.41 & $39 \%$ & 0.09 & $66 \%$ & 0.07 & $91 \%$ & 0.04 & $69 \%$ & 0.01 & $78 \%$ & 0.62 & $47 \%$ \\
\hline $\mathrm{H}$ - Transportation and Storage & 6.68 & $47 \%$ & 1.07 & $96 \%$ & 0.91 & $98 \%$ & 0.40 & $93 \%$ & 0.07 & $119 \%$ & 9.13 & $55 \%$ \\
\hline I- Accomodation and Food Service Activities & 17.53 & $41 \%$ & 0.88 & $54 \%$ & 0.37 & $62 \%$ & 0.09 & $71 \%$ & 0.02 & $89 \%$ & 18.89 & $42 \%$ \\
\hline $\mathrm{J}$ - Information and Communication & 6.35 & $34 \%$ & 0.50 & $94 \%$ & 0.39 & $111 \%$ & 0.21 & $87 \%$ & 0.05 & $94 \%$ & 7.50 & $38 \%$ \\
\hline L - Real Estate Activities & 5.76 & $23 \%$ & 0.13 & $108 \%$ & 0.12 & & 0.04 & & 0.01 & & 6.07 & $24 \%$ \\
\hline M-Professional, Scientific and Technical Activities & 23.40 & $28 \%$ & 1.20 & $112 \%$ & 0.71 & $128 \%$ & 0.38 & $152 \%$ & 0.10 & $213 \%$ & 25.78 & $30 \%$ \\
\hline $\mathrm{N}$ - Administrative and Support Service Activities & 8.04 & $33 \%$ & 0.71 & $107 \%$ & 0.67 & $118 \%$ & 0.30 & $83 \%$ & 0.11 & $106 \%$ & 9.84 & $37 \%$ \\
\hline Finland & & & & & & & & & & & & \\
\hline B - Mining and Quarrying & 0.34 & $43 \%$ & 0.03 & $57 \%$ & 0.02 & $69 \%$ & 0.01 & $82 \%$ & 0.00 & $100 \%$ & 0.40 & $45 \%$ \\
\hline C - Manufacturing & 9.83 & $52 \%$ & 1.57 & $91 \%$ & 1.34 & $101 \%$ & 0.84 & $98 \%$ & 0.21 & $91 \%$ & 13.79 & $60 \%$ \\
\hline D - Electricity, Gas, Steam and Air Conditioning Supply & 0.40 & $70 \%$ & 0.05 & $98 \%$ & 0.06 & $113 \%$ & 0.04 & $86 \%$ & 0.01 & $45 \%$ & 0.55 & $76 \%$ \\
\hline E - Water supply; Sewerage, Waste Management (...) & 0.91 & $78 \%$ & 0.07 & $71 \%$ & 0.04 & $83 \%$ & 0.01 & $40 \%$ & 0.00 & $75 \%$ & 1.04 & $77 \%$ \\
\hline $\mathrm{H}$ - Transportation and Storage & 6.63 & $30 \%$ & 0.73 & $86 \%$ & 0.39 & $89 \%$ & 0.20 & $113 \%$ & 0.04 & $79 \%$ & 7.99 & $34 \%$ \\
\hline I- Accomodation and Food Service Activities & 4.64 & $43 \%$ & 0.48 & $82 \%$ & 0.24 & $96 \%$ & 0.08 & $90 \%$ & 0.01 & $82 \%$ & 5.45 & $47 \%$ \\
\hline $\mathrm{J}$ - Information and Communication & 6.26 & $82 \%$ & 0.43 & $93 \%$ & 0.34 & $116 \%$ & 0.20 & $119 \%$ & 0.06 & $108 \%$ & 7.28 & $84 \%$ \\
\hline L - Real Estate Activities & 6.41 & $38 \%$ & 0.17 & $94 \%$ & 0.11 & $119 \%$ & 0.04 & $117 \%$ & 0.01 & $167 \%$ & 6.72 & $40 \%$ \\
\hline M-Professional, Scientific and Technical Activities & 19.71 & $62 \%$ & 0.94 & $120 \%$ & 0.56 & $112 \%$ & 0.24 & $134 \%$ & 0.04 & $124 \%$ & 21.48 & $65 \%$ \\
\hline $\mathrm{N}$ - Administrative and Support Service Activities & 5.02 & $43 \%$ & 0.54 & $86 \%$ & 0.45 & $101 \%$ & 0.23 & $100 \%$ & 0.05 & $88 \%$ & 6.28 & $48 \%$ \\
\hline France & & & & & & & & & & & & \\
\hline B - Mining and Quarrying & 1.11 & $83 \%$ & 0.29 & $127 \%$ & 0.24 & $124 \%$ & 0.09 & $123 \%$ & 0.02 & $173 \%$ & 1.74 & $94 \%$ \\
\hline C - Manufacturing & 166.46 & $96 \%$ & 17.24 & $120 \%$ & 12.43 & $115 \%$ & 6.65 & $107 \%$ & 1.71 & $107 \%$ & 204.48 & $99 \%$ \\
\hline D - Electricity, Gas, Steam and Air Conditioning Supply & 1.75 & $27 \%$ & 0.05 & $92 \%$ & 0.05 & $140 \%$ & 0.03 & $90 \%$ & 0.02 & $105 \%$ & 1.89 & $29 \%$ \\
\hline E - Water supply; Sewerage, Waste Management (...) & 4.85 & $90 \%$ & 0.57 & $118 \%$ & 0.47 & $113 \%$ & 0.29 & $109 \%$ & 0.10 & $114 \%$ & 6.28 & $95 \%$ \\
\hline $\mathrm{H}$ - Transportation and Storage & 78.03 & $104 \%$ & 5.02 & $97 \%$ & 4.16 & $119 \%$ & 1.98 & $114 \%$ & 0.40 & $124 \%$ & 89.58 & $104 \%$ \\
\hline I- Accomodation and Food Service Activities & 215.12 & $103 \%$ & 9.48 & $138 \%$ & 4.26 & $117 \%$ & 0.76 & $143 \%$ & 0.14 & $126 \%$ & 229.76 & $105 \%$ \\
\hline $\mathrm{J}$ - Information and Communication & 57.76 & $78 \%$ & 3.25 & $116 \%$ & 2.38 & $111 \%$ & 1.12 & $105 \%$ & 0.27 & $95 \%$ & 64.78 & $81 \%$ \\
\hline L - Real Estate Activities & 72.87 & $51 \%$ & 2.83 & $254 \%$ & 1.40 & $197 \%$ & 0.52 & $120 \%$ & 0.11 & $115 \%$ & 77.72 & $53 \%$ \\
\hline M-Professional, Scientific and Technical Activities & 155.99 & $53 \%$ & 7.62 & $98 \%$ & 4.15 & $100 \%$ & 1.68 & $106 \%$ & 0.37 & $108 \%$ & 169.80 & $56 \%$ \\
\hline $\mathrm{N}$ - Administrative and Support Service Activities & 74.59 & $60 \%$ & 4.99 & $110 \%$ & 3.95 & $118 \%$ & 2.41 & $150 \%$ & 0.47 & $137 \%$ & 86.40 & $64 \%$ \\
\hline Germany & & & & & & & & & & & & \\
\hline B - Mining and Quarrying & 0.46 & $50 \%$ & 0.12 & $33 \%$ & 0.11 & $47 \%$ & 0.08 & $74 \%$ & 0.02 & $86 \%$ & 0.80 & $48 \%$ \\
\hline C - Manufacturing & 37.87 & $35 \%$ & 9.79 & $28 \%$ & 9.99 & $62 \%$ & 13.65 & $85 \%$ & 3.83 & $97 \%$ & 75.12 & $42 \%$ \\
\hline D - Electricity, Gas, Steam and Air Conditioning Supply & 6.52 & $1046 \%$ & 0.22 & $138 \%$ & 0.36 & $119 \%$ & 0.40 & $96 \%$ & 0.13 & $78 \%$ & 7.63 & $456 \%$ \\
\hline E - Water supply; Sewerage, Waste Management (...) & 2.30 & $108 \%$ & 0.53 & $58 \%$ & 0.61 & $72 \%$ & 0.56 & $94 \%$ & 0.11 & $99 \%$ & 4.11 & $89 \%$ \\
\hline $\mathrm{H}$ - Transportation and Storage & 17.68 & $28 \%$ & 3.67 & $32 \%$ & 3.04 & $37 \%$ & 2.34 & $62 \%$ & 0.56 & $79 \%$ & 27.28 & $31 \%$ \\
\hline I - Accomodation and Food Service Activities & 12.90 & $8 \%$ & 2.38 & $9 \%$ & 1.98 & $14 \%$ & 1.03 & $25 \%$ & 0.18 & $72 \%$ & 18.46 & $8 \%$ \\
\hline $\mathrm{J}$ - Information and Communication & 24.23 & $34 \%$ & 1.94 & $36 \%$ & 1.66 & $46 \%$ & 1.58 & $73 \%$ & 0.39 & $94 \%$ & 29.80 & $36 \%$ \\
\hline L - Real Estate Activities & 39.24 & $23 \%$ & 1.48 & $59 \%$ & 1.13 & $86 \%$ & 0.75 & $145 \%$ & 0.23 & $315 \%$ & 42.82 & $25 \%$ \\
\hline M-Professional, Scientific and Technical Activities & 74.69 & $25 \%$ & 4.88 & $22 \%$ & 3.69 & $43 \%$ & 3.29 & $117 \%$ & 1.85 & $416 \%$ & 88.41 & $26 \%$ \\
\hline $\mathrm{N}$ - Administrative and Support Service Activities & 39.83 & $40 \%$ & 3.05 & $33 \%$ & 2.94 & $38 \%$ & 2.85 & $50 \%$ & 1.16 & $77 \%$ & 49.84 & $40 \%$ \\
\hline Ireland & & & & & & & & & & & & \\
\hline B - Mining and Quarrying & 0.02 & $27 \%$ & 0.01 & $30 \%$ & 0.01 & $40 \%$ & 0.01 & $73 \%$ & 0.00 & $75 \%$ & 0.06 & $34 \%$ \\
\hline C - Manufacturing & 1.09 & $49 \%$ & 0.50 & $55 \%$ & 0.46 & $65 \%$ & 0.27 & $53 \%$ & 0.06 & $42 \%$ & 2.38 & $53 \%$ \\
\hline D - Electricity, Gas, Steam and Air Conditioning Supply & 0.02 & $17 \%$ & 0.01 & & 0.00 & & 0.00 & $25 \%$ & 0.00 & & 0.03 & $22 \%$ \\
\hline E - Water supply; Sewerage, Waste Management (...) & 0.05 & $33 \%$ & 0.02 & $33 \%$ & 0.01 & $32 \%$ & 0.00 & $13 \%$ & 0.00 & $0 \%$ & 0.08 & $31 \%$ \\
\hline $\mathrm{H}$ - Transportation and Storage & 0.18 & $2 \%$ & 0.09 & $15 \%$ & 0.07 & $28 \%$ & 0.03 & $23 \%$ & 0.01 & $39 \%$ & 0.38 & $4 \%$ \\
\hline I- Accomodation and Food Service Activities & 0.11 & $1 \%$ & 0.06 & $4 \%$ & 0.09 & $10 \%$ & 0.10 & $19 \%$ & 0.01 & $38 \%$ & 0.37 & $2 \%$ \\
\hline $\mathrm{J}$ - Information and Communication & 0.55 & $8 \%$ & 0.17 & $49 \%$ & 0.10 & $40 \%$ & 0.07 & & 0.01 & & 0.89 & $12 \%$ \\
\hline L - Real Estate Activities & 0.21 & $3 \%$ & 0.04 & $29 \%$ & 0.02 & $26 \%$ & 0.02 & & 0.01 & & 0.29 & $4 \%$ \\
\hline M- Professional, Scientific and Technical Activities & 0.60 & $2 \%$ & 0.16 & $15 \%$ & 0.11 & $26 \%$ & 0.05 & $27 \%$ & 0.01 & $29 \%$ & 0.92 & $4 \%$ \\
\hline $\mathrm{N}$ - Adminis trative and Support Service Activities & 0.99 & $12 \%$ & 0.31 & $59 \%$ & 0.21 & $69 \%$ & 0.12 & $59 \%$ & 0.03 & $40 \%$ & 1.66 & $17 \%$ \\
\hline
\end{tabular}

Note: Number of enterprises in thousands. Data refer to the year 2009, or closest available years (for the number of employees). Source: OECD, calculations based on OECD-ORBIS@ June 2011 and Eurostat, Structural Business Statistics, extracted in October 2012. 
DSTI/DOC(2013)5

Table 3b. Firms by size class and industry - comparing ORBIS $\odot$ and structural business statistics data, 2009

\begin{tabular}{|c|c|c|c|c|c|c|c|c|c|c|c|c|}
\hline \multirow[b]{2}{*}{ Nace, rev. 2} & \multicolumn{2}{|c|}{0 to 9} & \multicolumn{2}{|c|}{10 to 19} & \multicolumn{2}{|c|}{20 to 49} & \multicolumn{2}{|c|}{50 to 249} & \multicolumn{2}{|c|}{$250+$} & \multicolumn{2}{|c|}{ All firms* } \\
\hline & $\begin{array}{c}\text { Firms } \\
\text { in ORBIS }\end{array}$ & $\begin{array}{c}\% \\
\text { of SBS }\end{array}$ & $\begin{array}{c}\text { Firms } \\
\text { in ORBIS }\end{array}$ & $\begin{array}{c}\% \\
\text { of SBS }\end{array}$ & $\begin{array}{c}\text { Firms } \\
\text { in ORBIS }\end{array}$ & \begin{tabular}{c|}
$\%$ \\
of SBS
\end{tabular} & $\begin{array}{c}\text { Firms } \\
\text { in ORBIS }\end{array}$ & \begin{tabular}{c|}
$\%$ \\
of SBS
\end{tabular} & $\begin{array}{c}\text { Firms } \\
\text { in ORBIS }\end{array}$ & $\begin{array}{c}\% \\
\text { of SBS }\end{array}$ & $\begin{array}{c}\text { Firms } \\
\text { in ORBIS }\end{array}$ & $\begin{array}{c}\% \\
\text { of SBS }\end{array}$ \\
\hline \multicolumn{13}{|l|}{ Italy } \\
\hline B - Mining and Quarrying & 1.16 & $60 \%$ & 0.36 & $85 \%$ & 0.19 & $112 \%$ & 0.07 & & 0.00 & & 1.77 & $69 \%$ \\
\hline C - Manufacturing & 86.84 & $24 \%$ & 27.08 & $58 \%$ & 18.20 & $82 \%$ & 9.82 & $106 \%$ & 1.40 & $104 \%$ & 143.35 & $33 \%$ \\
\hline D - Electricity, Gas, Steam and Air Conditioning Supply & 1.13 & $46 \%$ & 0.16 & $81 \%$ & 0.14 & $103 \%$ & 0.12 & $120 \%$ & 0.04 & $105 \%$ & 1.60 & $55 \%$ \\
\hline E - Water supply; Sewerage, Waste Management (...) & 2.57 & $43 \%$ & 0.76 & $63 \%$ & 0.53 & $79 \%$ & 0.39 & & 0.10 & & 4.34 & $51 \%$ \\
\hline H - Transportation and Storage & 21.05 & $17 \%$ & 4.86 & $67 \%$ & 3.21 & $89 \%$ & 1.74 & $100 \%$ & 0.35 & $105 \%$ & 31.22 & $23 \%$ \\
\hline I- Accomodation and Food Service Activities & 32.79 & $12 \%$ & 5.84 & $41 \%$ & 2.55 & $77 \%$ & 0.82 & $106 \%$ & 0.10 & $79 \%$ & 42.09 & $14 \%$ \\
\hline $\mathrm{J}$ - Information and Communication & 29.48 & $31 \%$ & 3.26 & $82 \%$ & 1.58 & $101 \%$ & 0.85 & $107 \%$ & 0.19 & $108 \%$ & 35.35 & $35 \%$ \\
\hline L - Real Es tate Activities & 33.72 & $16 \%$ & 1.40 & $257 \%$ & 0.67 & & 0.38 & $747 \%$ & 0.10 & & 36.27 & $17 \%$ \\
\hline M-Professional, Scientific and Technical Activities & 41.24 & $6 \%$ & 3.55 & $64 \%$ & 1.77 & $109 \%$ & 1.06 & $146 \%$ & 0.42 & $368 \%$ & 48.04 & $7 \%$ \\
\hline $\mathrm{N}$ - Administrative and Support Service Activities & 25.06 & $18 \%$ & 3.80 & $66 \%$ & 2.68 & $85 \%$ & 1.84 & $104 \%$ & 0.43 & $96 \%$ & 33.81 & $23 \%$ \\
\hline \multicolumn{13}{|l|}{ Netherlands } \\
\hline B - Mining and Quarrying & 0.26 & $113 \%$ & 0.04 & $125 \%$ & 0.03 & $148 \%$ & 0.03 & $108 \%$ & 0.02 & $300 \%$ & 0.38 & $121 \%$ \\
\hline C - Manufacturing & 14.90 & $42 \%$ & 4.50 & $111 \%$ & 3.94 & $114 \%$ & 1.91 & $88 \%$ & 0.43 & $113 \%$ & 25.68 & $56 \%$ \\
\hline D - Electricity, Gas, Steam and Air Conditioning Supply & 0.41 & $67 \%$ & 0.02 & $153 \%$ & 0.02 & $106 \%$ & 0.03 & $127 \%$ & 0.02 & $129 \%$ & 0.50 & $73 \%$ \\
\hline E - Water supply; Sewerage, Waste Management (...) & 0.90 & $101 \%$ & 0.20 & $135 \%$ & 0.15 & $119 \%$ & 0.09 & $96 \%$ & 0.02 & $73 \%$ & 1.35 & $106 \%$ \\
\hline $\mathrm{H}$ - Transportation and Storage & 8.29 & $38 \%$ & 1.98 & $111 \%$ & 1.70 & $115 \%$ & 0.95 & $116 \%$ & 0.15 & $117 \%$ & 13.05 & $50 \%$ \\
\hline I- Accomodation and Food Service Activities & 5.46 & $16 \%$ & 1.83 & $64 \%$ & 1.35 & $120 \%$ & 0.37 & $150 \%$ & 0.04 & $106 \%$ & 9.05 & $23 \%$ \\
\hline $\mathrm{J}$ - Information and Communication & 15.06 & $52 \%$ & 1.57 & $126 \%$ & 1.01 & $105 \%$ & 0.44 & $97 \%$ & 0.11 & $95 \%$ & 18.20 & $57 \%$ \\
\hline L - Real Estate Activities & 15.88 & $86 \%$ & 0.77 & $140 \%$ & 0.53 & $167 \%$ & 0.26 & $125 \%$ & 0.04 & $145 \%$ & 17.48 & $89 \%$ \\
\hline M-Professional, Scientific and Technical Activities & 78.72 & $59 \%$ & 4.84 & $121 \%$ & 2.98 & $133 \%$ & 1.11 & $133 \%$ & 0.25 & $175 \%$ & 87.90 & $62 \%$ \\
\hline $\mathrm{N}$ - Administrative and Support Service Activities & 14.43 & $47 \%$ & 1.96 & $85 \%$ & 1.71 & $82 \%$ & 0.93 & $71 \%$ & 0.16 & $63 \%$ & 19.18 & $53 \%$ \\
\hline Spain & & & & & & & & & & & & \\
\hline B - Mining and Quarrying & 1.55 & $84 \%$ & 0.50 & $134 \%$ & 0.35 & $148 \%$ & 0.12 & $149 \%$ & 0.02 & $160 \%$ & 2.53 & $99 \%$ \\
\hline C - Manufacturing & 74.27 & $48 \%$ & 18.50 & $104 \%$ & 12.99 & $102 \%$ & 5.19 & $105 \%$ & 0.88 & $101 \%$ & 111.83 & $58 \%$ \\
\hline D - Electricity, Gas, Steam and Air Conditioning Supply & 2.07 & $16 \%$ & 0.20 & $237 \%$ & 0.10 & $192 \%$ & 0.05 & $155 \%$ & 0.03 & $133 \%$ & 2.45 & $19 \%$ \\
\hline E - Water supply; Sewerage, Waste Management (...) & 1.47 & $45 \%$ & 0.31 & $79 \%$ & 0.25 & $79 \%$ & 0.15 & $71 \%$ & 0.05 & $88 \%$ & 2.24 & $52 \%$ \\
\hline $\mathrm{H}$ - Transportation and Storage & 23.71 & $11 \%$ & 4.85 & $85 \%$ & 2.96 & $92 \%$ & 1.10 & $94 \%$ & 0.18 & $99 \%$ & 32.79 & $15 \%$ \\
\hline I- Accomodation and Food Service Activities & 39.84 & $15 \%$ & 7.23 & $68 \%$ & 3.86 & $91 \%$ & 1.26 & $89 \%$ & 0.20 & $99 \%$ & 52.38 & $18 \%$ \\
\hline $\mathrm{J}$ - Information and Communication & 17.69 & $40 \%$ & 2.15 & $101 \%$ & 1.53 & $108 \%$ & 0.92 & $117 \%$ & 0.23 & $126 \%$ & 22.52 & $47 \%$ \\
\hline L - Real Estate Activities & 46.45 & $39 \%$ & 0.95 & $127 \%$ & 0.40 & $114 \%$ & 0.11 & $129 \%$ & 0.02 & $200 \%$ & 47.93 & $40 \%$ \\
\hline M-Professional, Scientific and Technical Activities & 70.11 & $20 \%$ & 5.38 & $75 \%$ & 2.75 & $101 \%$ & 1.24 & $111 \%$ & 0.28 & $143 \%$ & 79.76 & $22 \%$ \\
\hline $\mathrm{N}$ - Administrative and Support Service Activities & 21.84 & $21 \%$ & 3.02 & $63 \%$ & 2.39 & $75 \%$ & 1.53 & $80 \%$ & 0.48 & $91 \%$ & 29.25 & $25 \%$ \\
\hline Norway & & & & & & & & & & & & \\
\hline B - Mining and Quarrying & 0.86 & $107 \%$ & 0.08 & $98 \%$ & 0.06 & $85 \%$ & 0.05 & $94 \%$ & 0.04 & $100 \%$ & 1.08 & $104 \%$ \\
\hline C - Manufacturing & 8.54 & $62 \%$ & 1.44 & $97 \%$ & 1.17 & $103 \%$ & 0.67 & $102 \%$ & 0.12 & $101 \%$ & 11.93 & $69 \%$ \\
\hline D - Electricity, Gas, Steam and Air Conditioning Supply & 1.03 & $138 \%$ & 0.07 & $96 \%$ & 0.09 & $100 \%$ & 0.05 & $84 \%$ & 0.01 & $129 \%$ & 1.26 & $128 \%$ \\
\hline E - Water supply; Sewerage, Waste Management (...) & 0.52 & $60 \%$ & 0.09 & $107 \%$ & 0.08 & $118 \%$ & 0.03 & $125 \%$ & 0.00 & $133 \%$ & 0.73 & $69 \%$ \\
\hline $\mathrm{H}$ - Transportation and Storage & 7.87 & $39 \%$ & 0.62 & $81 \%$ & 0.44 & $105 \%$ & 0.21 & $98 \%$ & 0.07 & $93 \%$ & 9.21 & $42 \%$ \\
\hline I- Accomodation and Food Service Activities & 4.70 & $55 \%$ & 1.08 & $85 \%$ & 0.60 & $94 \%$ & 0.19 & $104 \%$ & 0.02 & $95 \%$ & 6.58 & $62 \%$ \\
\hline $\mathrm{J}$ - Information and Communication & 8.41 & $63 \%$ & 0.57 & $105 \%$ & 0.36 & $100 \%$ & 0.22 & $100 \%$ & 0.04 & $106 \%$ & 9.60 & $66 \%$ \\
\hline L - Real Estate Activities & 59.93 & $140 \%$ & 0.37 & $197 \%$ & 0.14 & $280 \%$ & 0.06 & $278 \%$ & 0.01 & $400 \%$ & 60.52 & $140 \%$ \\
\hline M-Professional, Scientific and Technical Activities & 25.67 & $68 \%$ & 1.12 & $107 \%$ & 0.50 & $108 \%$ & 0.19 & $98 \%$ & 0.03 & $115 \%$ & 27.50 & $70 \%$ \\
\hline $\mathrm{N}$ - Administrative and Support Service Activities & 9.83 & $65 \%$ & 0.64 & $101 \%$ & 0.41 & $103 \%$ & 0.28 & $110 \%$ & 0.06 & $108 \%$ & 11.21 & $68 \%$ \\
\hline Sweden & & & & & & & & & & & & \\
\hline B - Mining and Quarrying & 0.59 & $94 \%$ & 0.04 & $86 \%$ & 0.02 & $100 \%$ & 0.01 & $129 \%$ & 0.00 & $40 \%$ & 0.65 & $94 \%$ \\
\hline C - Manufacturing & 43.40 & $92 \%$ & 2.94 & $99 \%$ & 2.06 & $96 \%$ & 1.27 & $89 \%$ & 0.30 & $82 \%$ & 49.97 & $93 \%$ \\
\hline D - Electricity, Gas, Steam and Air Conditioning Supply & 1.44 & $104 \%$ & 0.07 & $115 \%$ & 0.08 & $98 \%$ & 0.07 & $85 \%$ & 0.01 & $46 \%$ & 1.67 & $102 \%$ \\
\hline E - Water supply; Sewerage, Waste Management (...) & 0.98 & $107 \%$ & 0.09 & $97 \%$ & 0.07 & $124 \%$ & 0.05 & $96 \%$ & 0.01 & $133 \%$ & 1.19 & $106 \%$ \\
\hline $\mathrm{H}$ - Transportation and Storage & 26.79 & $102 \%$ & 1.43 & $98 \%$ & 0.75 & $96 \%$ & 0.35 & $97 \%$ & 0.09 & $98 \%$ & 29.40 & $102 \%$ \\
\hline I- Accomodation and Food Service Activities & 26.64 & $106 \%$ & 1.31 & $91 \%$ & 0.59 & $80 \%$ & 0.13 & $66 \%$ & 0.02 & $70 \%$ & 28.67 & $104 \%$ \\
\hline $\mathrm{J}$ - Information and Communication & 43.10 & $93 \%$ & 0.96 & $102 \%$ & 0.65 & $95 \%$ & 0.32 & $91 \%$ & 0.08 & $105 \%$ & 45.10 & $93 \%$ \\
\hline L - Real Estate Activities & 51.23 & $109 \%$ & 0.47 & $112 \%$ & 0.30 & $111 \%$ & 0.17 & $113 \%$ & 0.02 & $116 \%$ & 52.19 & $109 \%$ \\
\hline M-Professional, Scientific and Technical Activities & 138.37 & $97 \%$ & 1.72 & $101 \%$ & 0.96 & $101 \%$ & 0.40 & $106 \%$ & 0.08 & $133 \%$ & 141.52 & $97 \%$ \\
\hline $\mathrm{N}$ - Administrative and Support Service Activities & 27.36 & $98 \%$ & 1.01 & $103 \%$ & 0.63 & $93 \%$ & 0.34 & $100 \%$ & 0.09 & $88 \%$ & 29.42 & $98 \%$ \\
\hline Switzerland & & & & & & & & & & & & \\
\hline B - Mining and Quarrying & 0.12 & $142 \%$ & 0.12 & $252 \%$ & 0.06 & $115 \%$ & 0.03 & $176 \%$ & 0.00 & & 0.34 & $166 \%$ \\
\hline C - Manufacturing & 11.32 & $96 \%$ & 5.08 & $128 \%$ & 4.16 & $147 \%$ & 2.24 & $118 \%$ & 0.65 & $151 \%$ & 23.46 & $112 \%$ \\
\hline D - Electricity, Gas, Steam and Air Conditioning Supply & 0.16 & $180 \%$ & 0.08 & $96 \%$ & 0.17 & $184 \%$ & 0.07 & $109 \%$ & 0.03 & $143 \%$ & 0.51 & $145 \%$ \\
\hline E - Water supply; Sewerage, Waste Management (...) & 0.27 & $75 \%$ & 0.21 & $146 \%$ & 0.16 & $135 \%$ & 0.04 & $97 \%$ & 0.01 & $200 \%$ & 0.69 & $103 \%$ \\
\hline $\mathrm{H}$ - Transportation and Storage & 2.26 & $87 \%$ & 1.80 & $219 \%$ & 0.45 & $74 \%$ & 0.27 & $88 \%$ & 0.10 & $138 \%$ & 4.88 & $110 \%$ \\
\hline I- Accomodation and Food Service Activities & 6.91 & $48 \%$ & 1.37 & $42 \%$ & 0.71 & $56 \%$ & 0.33 & $79 \%$ & 0.06 & $141 \%$ & 9.39 & $49 \%$ \\
\hline $\mathrm{J}$ - Information and Communication & 9.82 & $269 \%$ & 1.41 & $156 \%$ & 0.74 & $131 \%$ & 0.37 & $145 \%$ & 0.07 & $151 \%$ & 12.40 & $229 \%$ \\
\hline L - Real Estate Activities & 7.62 & $476 \%$ & 0.71 & $273 \%$ & 0.33 & $230 \%$ & 0.15 & $339 \%$ & 0.03 & $550 \%$ & 8.84 & $430 \%$ \\
\hline M- Professional, Scientific and Technical Activities & 27.94 & $178 \%$ & 2.25 & $80 \%$ & 1.09 & $92 \%$ & 0.38 & $96 \%$ & 0.08 & $115 \%$ & 31.74 & $157 \%$ \\
\hline $\mathrm{N}$ - Adminis trative and Support Service Activities & 11.63 & $230 \%$ & 1.39 & $128 \%$ & 0.54 & $92 \%$ & 0.29 & $86 \%$ & 0.09 & $144 \%$ & 13.94 & $196 \%$ \\
\hline
\end{tabular}

Note: Number of enterprises in thousands. Data refer to the year 2009, or closest available years (for the number of employees). Source: OECD, calculations based on OECD-ORBIS $\odot$ June 2011 and Eurostat, Structural Business Statistics, extracted in October 2012. 
With respect to group structure and ownership links, the information contained in ORBISC encompasses parent companies (owners) and affiliates (subsidiaries), where applicable, with shareholders that can be firms or physical persons. Ownership linkages include direct and indirect links (up to the tenth level of ownership, both upward and downward) and are provided on a yearly basis. This should help capture the composition of groups and conglomerates at each point in time considered, and to follow their evolution. However, as information on ownership links is neither provided for all firms nor for all links belonging to a certain company, it is not always possible to fully appreciate the composition and structure of groups and holdings, and to estimate direct or total (indirect) ownership percentages.

Table $4 \mathrm{a}$ and Table $4 \mathrm{~b}$ show the number and share of firms in ORBIS $\odot$ for which information on ownership structure is available, as well as the number and percentage of firms being Global Ultimate Owners (GUOs) or Domestic Ultimate Owners (DUOs). Firms are bound to belong to a group when there is at least one shareholder that reports $25 \%$ or more of their ownership. It is conversely assumed - although only for illustrative purposes - that firms are independent in case no ownership link exists in the database. The number and share of firms for which ownership links exist but remain below $25 \%$ are not shown in Table $4 \mathrm{a}$ and Table $4 \mathrm{~b}$, as in the absence of further information about e.g. control rights, it is difficult to clearly tag them as being independent or part of a group.

Table 4a. Distribution of firms according to ownership structure, 2009 All firms (thousands)

\begin{tabular}{|c|c|c|c|c|c|c|c|c|c|}
\hline \multirow{2}{*}{\multicolumn{2}{|c|}{ All firms }} & \multicolumn{6}{|c|}{ Firms belonging to a group } & \multicolumn{2}{|c|}{ Independent firms } \\
\hline & & Firms & $\%$ of total & $\begin{array}{c}\text { of which } \\
\text { GUO }\end{array}$ & $\%$ & $\begin{array}{c}\text { of which } \\
\text { DUO }\end{array}$ & $\%$ & Firms & $\%$ of total \\
\hline Aus tria & 125.5 & 85.5 & $68.1 \%$ & 0.9 & $1.1 \%$ & 0.6 & $0.7 \%$ & 12.6 & $10.0 \%$ \\
\hline Belgium & 215.6 & 24.5 & $11.4 \%$ & 5.7 & $23.1 \%$ & 0.3 & $1.3 \%$ & 189.3 & $87.8 \%$ \\
\hline Canada & 860.0 & 79.4 & $9.2 \%$ & 23.1 & $29.1 \%$ & 1.7 & $2.1 \%$ & 779.7 & $90.7 \%$ \\
\hline Finland & 120.4 & 13.4 & $11.1 \%$ & 4.1 & $30.9 \%$ & 0.2 & $1.6 \%$ & 106.1 & $88.1 \%$ \\
\hline France & 1645.2 & 161.1 & $9.8 \%$ & 21.6 & $13.4 \%$ & 4.4 & $2.7 \%$ & 1473.7 & $89.6 \%$ \\
\hline Germany & 372.1 & 314.9 & $84.6 \%$ & 9.0 & $2.9 \%$ & 6.0 & $1.9 \%$ & 39.5 & $10.6 \%$ \\
\hline Ireland & 16.1 & 13.1 & $81.1 \%$ & 0.3 & $2.4 \%$ & 0.3 & $2.4 \%$ & 1.8 & $11.5 \%$ \\
\hline Italy & 619.4 & 409.5 & $66.1 \%$ & 8.5 & $2.1 \%$ & 11.3 & $2.8 \%$ & 198.3 & $32.0 \%$ \\
\hline Japan & 219.9 & 78.2 & $35.5 \%$ & 69.0 & $88.3 \%$ & 0.1 & $0.2 \%$ & 141.7 & $64.4 \%$ \\
\hline Netherlands & 339.1 & 21.6 & $6.4 \%$ & 0.3 & $1.4 \%$ & 0.0 & $0.2 \%$ & 317.5 & $93.6 \%$ \\
\hline Norway & 236.2 & 175.2 & $74.2 \%$ & 16.1 & $9.2 \%$ & 0.7 & $0.4 \%$ & 51.9 & $22.0 \%$ \\
\hline Spain & 586.3 & 190.7 & $32.5 \%$ & 12.2 & $6.4 \%$ & 1.3 & $0.7 \%$ & 323.9 & $55.3 \%$ \\
\hline Sweden & 780.9 & 79.8 & $10.2 \%$ & 24.7 & $31.0 \%$ & 0.5 & $0.7 \%$ & 698.8 & $89.5 \%$ \\
\hline Switzerland & 200.7 & 15.0 & $7.5 \%$ & 3.5 & $23.1 \%$ & 0.4 & $2.4 \%$ & 184.6 & $92.0 \%$ \\
\hline United States & 12380.3 & 790.6 & $6.4 \%$ & 111.0 & $14.0 \%$ & 2.7 & $0.3 \%$ & 9194.1 & $74.3 \%$ \\
\hline
\end{tabular}

Note: The identification of the group structure is based on information contained in the ORBIS@ ownership structure table for the year 2009. Firms are denoted as belonging to a group when one or more shareholders reporting at least $25 \%$ of ownership exist. Firms are denoted as independent when no information on possible shareholders exists in the ownership structure table. The number and the share of firms for which ownership links exist but remain below $25 \%$ are not shown. Ownership data relate to the year 2009 .

Source: OECD, calculations based on OECD-ORBIS 2011, October 2012. 
Table 4b. Ownership structure - firms with more than 20 employees (thousands), 2009

\begin{tabular}{|c|c|c|c|c|c|c|c|c|c|}
\hline \multirow{2}{*}{\multicolumn{2}{|c|}{$\begin{array}{l}\text { Firms with more } \\
\text { than } 20 \text { employees }\end{array}$}} & \multicolumn{6}{|c|}{ Firms belonging to a group } & \multicolumn{2}{|c|}{ Independent firms } \\
\hline & & Firms & $\%$ of total & $\begin{array}{c}\text { of which } \\
\text { GUO }\end{array}$ & $\%$ & $\begin{array}{c}\text { of which } \\
\text { DuO }\end{array}$ & $\%$ & Firms & $\%$ of total \\
\hline Austria & 16.0 & 14.4 & $90.0 \%$ & 0.4 & $2.5 \%$ & 0.2 & $1.7 \%$ & 0.7 & $4.5 \%$ \\
\hline Belgium & 16.5 & 6.8 & $41.6 \%$ & 1.1 & $16.1 \%$ & 0.2 & $3.4 \%$ & 9.2 & $55.8 \%$ \\
\hline Canada & 72.4 & 30.6 & $42.3 \%$ & 12.3 & $40.2 \%$ & 1.4 & $4.7 \%$ & 41.6 & $57.5 \%$ \\
\hline Finland & 8.3 & 4.3 & $52.0 \%$ & 1.3 & $29.2 \%$ & 0.2 & $3.7 \%$ & 3.8 & $45.2 \%$ \\
\hline France & 73.4 & 46.6 & $63.4 \%$ & 3.8 & $8.2 \%$ & 1.6 & $3.5 \%$ & 24.9 & $33.9 \%$ \\
\hline Germany & 79.6 & 74.1 & $93.1 \%$ & 5.6 & $7.6 \%$ & 3.5 & $4.8 \%$ & 3.4 & $4.3 \%$ \\
\hline Ireland & 4.0 & 3.1 & $77.6 \%$ & 0.2 & $6.6 \%$ & 0.2 & $6.4 \%$ & 0.4 & $9.9 \%$ \\
\hline Italy & 68.5 & 49.3 & $72.0 \%$ & 3.5 & $7.1 \%$ & 3.3 & $6.7 \%$ & 17.2 & $25.1 \%$ \\
\hline Japan & 65.3 & 49.5 & $75.9 \%$ & 41.8 & $84.4 \%$ & 0.1 & $0.3 \%$ & 15.7 & $24.1 \%$ \\
\hline Netherlands & 31.7 & 2.8 & $8.8 \%$ & 0.2 & $5.7 \%$ & 0.0 & $1.0 \%$ & 28.9 & $91.2 \%$ \\
\hline Norway & 11.6 & 10.0 & $85.9 \%$ & 1.3 & $13.2 \%$ & 0.3 & $2.7 \%$ & 1.2 & $10.1 \%$ \\
\hline Spain & 57.2 & 36.2 & $63.2 \%$ & 3.0 & $8.4 \%$ & 0.8 & $2.1 \%$ & 10.2 & $17.8 \%$ \\
\hline Sweden & 17.0 & 10.6 & $62.7 \%$ & 1.9 & $17.4 \%$ & 0.2 & $2.1 \%$ & 6.2 & $36.4 \%$ \\
\hline Switzerland & 23.4 & 6.9 & $29.7 \%$ & 1.4 & $20.6 \%$ & 0.2 & $3.5 \%$ & 16.0 & $68.5 \%$ \\
\hline United States & 616.8 & 281.9 & $45.7 \%$ & 70.1 & $24.8 \%$ & 2.4 & $0.9 \%$ & 288.9 & $46.8 \%$ \\
\hline
\end{tabular}

Note: Number of firms expressed in thousands. The identification of the group structure is based on information contained in the ORBISC ownership structure table for the year 2009. Firms are denoted as belonging to a group when one or more shareholders reporting at least $25 \%$ of ownership exist. Firms are denoted as independent when no information on possible shareholders exists in the ownership structure table. Ownership data relate to the year 2009.

Source: OECD, calculations based on OECD-ORBIS 2011, October 2012.

Table 4a refers to all firms, whereas Table $4 \mathrm{~b}$ relates to firms with 20 or more employees, with ownership structure referring to the year 2009. A comparison between Table $4 \mathrm{a}$ and Table $4 \mathrm{~b}$ seems to suggest that being part of a group is positively correlated with firm size, given that the proportion of firms belonging to a group is systematically higher when firms with 20 or more employees are considered. The opposite is seemingly true with respect to independent firms.

As the status of firms and their being independent, or otherwise belonging to a group, cannot be confidently established, in what follows we rely on statistics calculated at the unconsolidated level and attribute patents to the assignee indicated in the patent document. Although this implies overlooking the way in which belonging to a conglomerate or group may affect the innovative behaviour of firms, it nevertheless avoids interpreting missing data as zeros and assuming (possibly mistakenly) that firms for which no ownership infomation is available are independent.

Finally, the choice of countries on which the present analysis relies upon has been driven by the outcome of the pairing of firm data with patent data. The matching exercise carried out for the purpose is discussed in detail in the next section. The guiding principle in this case has been to identify those countries featuring the highest proportion of patents that are linked to ORBISC firms successfully. As the PATSTAT database is known to be comprehensive in its coverage, maximising the number of patents that are allocated to firms minimises the risk of mistakenly describing firms as non-patenting. Evidently, no analogous possibility exists with respect to those firms that do not patent, and it is not possible to establish the sample selection bias determined by the coverage of ORBISC .

\section{Patent data}

The OECD Patent database covers bibliographic records of patent applications filed at most Intellectual Property (IP) Offices worldwide. The information available covers three main categories: the technical description of the invention (title, technical classes, citations); the ownership of the invention (inventor and applicant/owner); and the history of the application (key administrative dates and legal steps), including patent renewal data related to EPO patents, where appropriate. The data contained in the OECD Patent database mostly derive from the Worldwide Patent Statistical Database, PATSTAT, 
maintained by the EPO, and relate to the April 2012 PATSTAT version. The information contained therein is complemented by: data from the EPO's Bibliographic Data (EPO online services), gathered in order to collect further details on patents applications filed to the EPO; data about filings made through the Patent Co-operation Treaty (PCT); and patent renewal data recorded in the Worldwide Legal Status Database maintained by the EPO.

The OECD patent database provides an exhaustive and comprehensive coverage of all patents filed at EPO and through the PCT system since 1978, as well as patents granted by the US Patent and Trademark Office (USPTO) since 1976. In the present study, patenting firms are defined as firms that have filed at least one patent application either at the EPO, the USPTO or through the PCT since late $1978 .{ }^{2}$ Patent renewal data conversely refer to granted patents recorded in the Worldwide Legal Status Database maintained by the EPO. More details about the patent-related information used for the analysis are supplied in later sections, when discussing the statistics presented.

As no numeric firm identifier is available in patent documents, firms have to be linked to patent assignees' names by means of string matching algorithms designed to optimise the precision of the match. Only countries for which the matching rate is above $80 \%$ of patents in the late 2000 s are considered in the sample (i.e. Austria, Belgium, Canada, Switzerland, Germany, Spain, Finland, France, Ireland, Italy, Japan, Netherlands, Norway, Sweden, United States).

\section{Linking patent applicant names to firm names}

Characterising the patenting behaviour of firms of different ages and sizes requires linking patent data to enterprise data. To this end, patent assignees' names available in PATSTAT have been matched to the names of the firms included in the OECD-ORBIS 2011 database. The linking has been carried out on a bycountry basis using a series of algorithms contained in the Imalinker system (Idener Multi Algorithm Linker) developed for the OECD by IDENER, Seville, 2011. The matching exercise carried out, which allows aligning information on patenting activities with those related to the characteristics and performance of firms, is implemented over a number of key steps:

- The names of firms included in the patent and the ORBIS $\odot$ databases are separately harmonised using country-specific "dictionaries", aimed at dealing with legal entities denomination (e.g. 'Limited" and 'Ltd'), common names and expressions, as well as phonetic and linguistic rules, which may affect how enterprise names are written. Compiling suitable country and language-specific dictionaries requires specific country level knowledge, but it helps to effectively reduce spelling variations of a same company name.

- A series of string-matching algorithms - mainly token-based and string-metric-based, like token frequency matching, Levensthein (1965) and Jaro-Winkler (Winkler, 1999) distances - then compares the harmonised names from the two datasets and provides a matching accuracy score for each pair. The precision of the match, which depends on minimising the number of false positive matches, is ensured through a selection of pairs of patenting firm/company names made on the basis of high-score thresholds imposed on the algorithm.

- A post processing stage is handled manually and requires reviewing the results of the matches; assessing the proportion of non-matched patenting firms (false negatives) within patenting firms; and identifying new matches either on a case by case basis (e.g. allowing for lower thresholds for a given algorithm) or by correcting and augmenting dictionaries.

2. More information can be found at www.oecd.org/sti/ipr-statistics. 
The matching exercise does not systematically result in unique pairs of names, as in many cases a patenting firm may correspond to several entries in OECD-ORBIS 2011. This is due to a feature of this database, in which multiple entries for the same name may depend on issues as the existence of branches or affiliates having the very same company name. As a consequence, further filtering and consolidation are required, using the information contained in additional fields like the address of the company and the company's ownership structure. To this end, a series of disambiguation rules has been followed and choices made in order to reduce the number of multiple matches. These for instance include removing the links to the branches and using data related to the Domestic Ultimate Owner when available (instead of the other possible matched names). Following Bureau Van Dijk's suggestion, in case of multiple matches, patents are attributed to the applicant name providing the largest turnover figure. Finally, links to records for which neither turnover nor number of employees are reported are removed.

The disambiguation rules used allow us to significantly reduce the number of firms for which multiple matches had been initially identified. Figure 5 shows the overall matching rate in terms of number of patent owners, for the countries considered. It further shows the share of unique matches obtained before and after the disambiguation process, as well as the share of multiple matches initially identified and those that remain after the additional cleaning performed. In the case of countries like Japan, the United States and France the disambiguation procedure substantially increases the ability to uniquely identify patent assignee firms. This contributes to reduce the possible selection bias that may be determined by the inability to uniquely assign such IP rights and by leaving (a big number of) patenting firms off the analysis.

Table 5. Impact of the disambiguation on the matching results, by country

Share of PATSTAT's applicant names matched to OECD-ORBIS 2011 as a percentage of total applicants

\begin{tabular}{lc|rr|rr}
\hline \multirow{2}{*}{$\%$} & \multirow{2}{*}{$\begin{array}{c}\text { Overall } \\
\text { matching }\end{array}$} & \multicolumn{2}{|c|}{ Unique match } & \multicolumn{2}{c}{ Multiple match } \\
\cline { 3 - 6 } & rate & Before & After & \multicolumn{2}{c}{ Disambiguation } \\
& 41.9 & 55.1 & 94.0 & 44.9 & Bfter \\
\hline Austria & 50.6 & 72.8 & 99.1 & 27.2 & 0.9 \\
Belgium & 40.7 & 66.1 & 98.5 & 33.9 & 1.5 \\
Canada & 44.9 & 91.7 & 99.7 & 8.3 & 0.3 \\
Finland & 58.0 & 47.5 & 92.8 & 52.5 & 7.2 \\
France & 33.3 & 66.4 & 99.6 & 33.6 & 0.4 \\
Germany & 51.0 & 96.9 & 100.0 & 3.1 & 0.0 \\
Ireland & 34.1 & 72.3 & 99.7 & 27.7 & 0.3 \\
Italy & 68.5 & 20.6 & 98.0 & 79.4 & 2.0 \\
Japan & 59.3 & 74.0 & 96.7 & 26.0 & 3.3 \\
Netherlands & 54.5 & 78.7 & 97.7 & 21.3 & 2.3 \\
Norway & 31.6 & 62.3 & 99.5 & 37.7 & 0.5 \\
Spain & 37.5 & 84.8 & 99.8 & 15.2 & 0.2 \\
Sweden & 31.8 & 90.7 & 99.9 & 9.3 & 0.1 \\
Switzerland & 66.8 & 48.4 & 99.5 & 51.6 & 0.5 \\
United States & & \multicolumn{3}{c}{}
\end{tabular}

Note: Matching rates are based on the whole population of applicants provided in PATSTAT database.

Source: OECD, calculations based on EPO, Worldwide Patent Statistical Database, April 2012 and OECD-ORBIS 2011, October 2012.

\section{Results of the matching and data used for the analysis}

The coverage of OECD-ORBIS 2011 leads to matching rates that are highest for the period 2000-10. These nevertheless appear heterogeneous across countries and over time, with small firms that are typically under-represented. To partially address this and other selection and data consistency concerns, we restrict the analysis to those countries with matching rates around $70 \%$ or higher on average in terms of applicant names and around $80 \%$ or above on average in terms of patents filed during the years considered. Although countries like the United Kingdom have a relatively high overall matching rate, the lack of the additional 
information needed to disambiguate patent assignees' names makes it impossible to include them in the analysis. The analysis is thus restricted to Austria, Belgium, Canada, Finland, France, Germany, Ireland, Italy, Japan, Netherlands, Norway, Spain, Sweden, Switzerland, and the United States.

Table 6 shows the percentage of patent assignees matched to the firms in ORBISC ${ }^{\circ}$, as well as the share of patents applications matched. Coverage appears best for the Netherlands ( $85 \%$ on average) and Japan (84.4\% on average) in terms of patent owners, as well as in terms of patents filed (respectively $95 \%$ and $94 \%$, on average). Among the countries considered, Canada features the lowest share of patenting companies matched (66\% on average), whereas Italy features relatively lower matches in terms of patents linked ( $78 \%$ on average).

Table 6. Matching rates, by country

Share of matched applicant names in total applicants, and share of matched patents in total patents Patent applications filed at EPO, USPTO or through the PCT

\begin{tabular}{lrllll|lllll|}
\hline & \multicolumn{5}{c|}{ Applicants (\%) } & \multicolumn{5}{c|}{ Patent filings (\%) } \\
\cline { 2 - 12 } & $\mathbf{1 9 9 9}$ & $\mathbf{2 0 0 2}$ & $\mathbf{2 0 0 5}$ & $\mathbf{2 0 0 8}$ & $\mathbf{2 0 1 0}$ & $\mathbf{1 9 9 9}$ & $\mathbf{2 0 0 2}$ & $\mathbf{2 0 0 5}$ & $\mathbf{2 0 0 8}$ & $\mathbf{2 0 1 0}$ \\
\hline Austria & 64.5 & 72.9 & 82.9 & 85.9 & 90.2 & 66.3 & 82.4 & 89.3 & 90.5 & 95.0 \\
Belgium & 70.3 & 71.2 & 79.9 & 82.4 & 82.3 & 80.9 & 82.4 & 87.3 & 88.4 & 84.5 \\
Canada & 52.0 & 60.4 & 73.7 & 72.9 & 72.2 & 70.5 & 74.2 & 83.8 & 84.0 & 85.3 \\
Finland & 63.5 & 73.1 & 81.4 & 81.5 & 83.4 & 75.3 & 86.5 & 89.4 & 92.3 & 87.3 \\
France & 76.9 & 80.5 & 83.4 & 84.6 & 88.0 & 84.9 & 89.6 & 91.6 & 93.4 & 93.0 \\
Germany & 66.4 & 74.9 & 79.7 & 83.5 & 86.8 & 80.1 & 86.1 & 88.5 & 92.1 & 92.1 \\
Ireland & 64.7 & 78.3 & 85.3 & 85.1 & 88.7 & 70.6 & 85.6 & 92.3 & 90.8 & 93.2 \\
Italy & 64.6 & 69.0 & 73.4 & 76.0 & 76.1 & 72.2 & 75.7 & 79.4 & 80.9 & 82.7 \\
Japan & 79.3 & 80.8 & 84.7 & 88.0 & 89.1 & 90.3 & 91.1 & 93.0 & 97.3 & 98.5 \\
Netherlands & 79.1 & 81.4 & 85.9 & 89.3 & 90.4 & 90.5 & 94.3 & 97.1 & 97.1 & 97.0 \\
Norway & 76.4 & 79.5 & 85.0 & 87.8 & 88.5 & 85.9 & 82.1 & 83.3 & 90.5 & 87.9 \\
Spain & 70.8 & 73.4 & 78.2 & 80.4 & 79.4 & 73.9 & 79.3 & 81.7 & 82.0 & 78.8 \\
Sweden & 69.2 & 74.7 & 84.8 & 88.6 & 88.4 & 83.7 & 87.6 & 93.6 & 96.4 & 94.5 \\
Switzerland & 70.0 & 72.4 & 81.0 & 83.8 & 86.7 & 75.3 & 77.6 & 83.1 & 86.3 & 88.2 \\
United States & 74.7 & 77.2 & 84.8 & 85.7 & 86.4 & 85.8 & 86.7 & 89.7 & 88.1 & 91.2 \\
\hline
\end{tabular}

Source: OECD, calculations based on EPO, Worldwide Patent Statistical Database, April 2012 and OECD-ORBIS 2011, October 2012.

\section{Characterising the patenting activities of firms}

\section{Patenting and non-patenting firms}

In what follows, a number of descriptive statistics aim to characterise the patenting behaviour of firms and to describe the possible differences that might exist between these types of innovators and those firms that do not rely on this intellectual property right.

Table 7 and Figure 1 show the absolute number of patenting and non patenting firms by country, as well as the share of the relevant population that these numbers correspond to. In line with previous studies, patenting firms are found to represent a small part of the population of firms, i.e. between $1.6 \%$ in Ireland and $8.8 \%$ in Germany. Consequently, the proportion of firms that do not rely on patents is constantly above $90 \%$ of the total number of firms included in the study. Accounting for possible group structures may likely lead to different results, but such a feature cannot be properly addressed here due to the consolidation-related issues mentioned before. 
Table 7. Patenting and non-patenting firms, by country, 2009

\begin{tabular}{lr|rr|rr}
\hline & Total firms & \multicolumn{2}{|c|}{ Patenting } & \multicolumn{2}{|c}{ Non-patenting firm } \\
\hline Austria & 21.1 & 1.1 & $5.2 \%$ & 20.0 & $94.8 \%$ \\
Belgium & 18.1 & 0.7 & $3.9 \%$ & 17.3 & $96.1 \%$ \\
Canada & 142.1 & 2.4 & $1.7 \%$ & 139.7 & $98.3 \%$ \\
Finland & 9.5 & 0.7 & $7.2 \%$ & 8.8 & $92.8 \%$ \\
France & 94.8 & 4.3 & $4.6 \%$ & 90.5 & $95.4 \%$ \\
Germany & 106.0 & 9.3 & $8.8 \%$ & 96.7 & $91.2 \%$ \\
Ireland & 7.2 & 0.1 & $1.6 \%$ & 7.1 & $98.4 \%$ \\
Italy & 80.2 & 4.8 & $6.0 \%$ & 75.4 & $94.0 \%$ \\
Japan & 92.1 & 5.2 & $5.6 \%$ & 86.9 & $94.4 \%$ \\
Netherlands & 42.9 & 1.3 & $2.9 \%$ & 41.7 & $97.1 \%$ \\
Norway & 13.1 & 0.4 & $3.1 \%$ & 12.7 & $96.9 \%$ \\
Spain & 79.9 & 1.5 & $1.9 \%$ & 78.4 & $98.1 \%$ \\
Sweden & 18.9 & 1.1 & $5.7 \%$ & 17.9 & $94.3 \%$ \\
Switzerland & 25.1 & 2.1 & $8.5 \%$ & 23.0 & $91.5 \%$ \\
United States & 1167.5 & 28.9 & $2.5 \%$ & 1138.7 & $97.5 \%$ \\
\hline
\end{tabular}

Note: Number of firms expressed in thousands. Firms reporting 20 or more employees in OECD-ORBIS 2011.

Source: OECD calculations based on EPO Worldwide Patent Statistical Database (April 2012) and OECD-ORBIS 2011, October 2012.

Figure 1. Share of patenting firms in total firms, by country, 2009

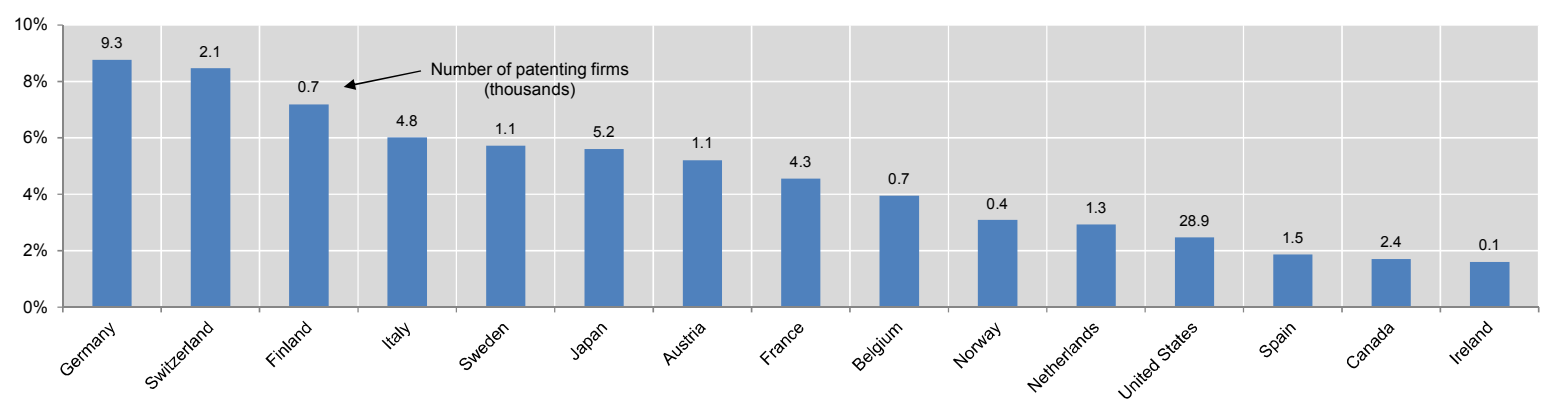

Note: Firms reporting 20 or more employees in OECD-ORBIS 2011.

Source: OECD calculations based on EPO Worldwide Patent Statistical Database (April 2012) and OECD-ORBIS 2011, October 2012.

Figure 1 ranks countries on the basis of their proportion of patenting firms. This rank would look different if countries were to be ranked on the basis of the absolute number of firms being the assignees of one of more patents. In this case, the rank would see the United States first, followed by Germany and then Japan, as it can be inferred from the small numbers put on the top of the bars, as well as the figures shown in Table 7.

\section{Patenting activity by firm size}

Figure 2 shows the way patenting activities are distributed across firms of different size, as well as the distribution by class size of those firms that do not rely on patents. To this end, and to account for the different ways in which small and medium enterprises (SMEs) are defined in Europe and in the United States, ${ }^{3}$ patenting firms are subdivided into four size classes: 20-49 employees, 50-249 employees, 250499, and firms having 500 or more employees. In general, medium and big firms account for the majority of patenting firms, although differences exist across countries with respect to the proportion of small, medium and big firms that patent and those that do not.

3. In Europe SMEs are defined as firms with less than 250 employees, whereas in the United States the upper limit for SMEs is set to 500 employees. 
Figure 2. Patenting firms and non-patenting firms by employee size class (2009)

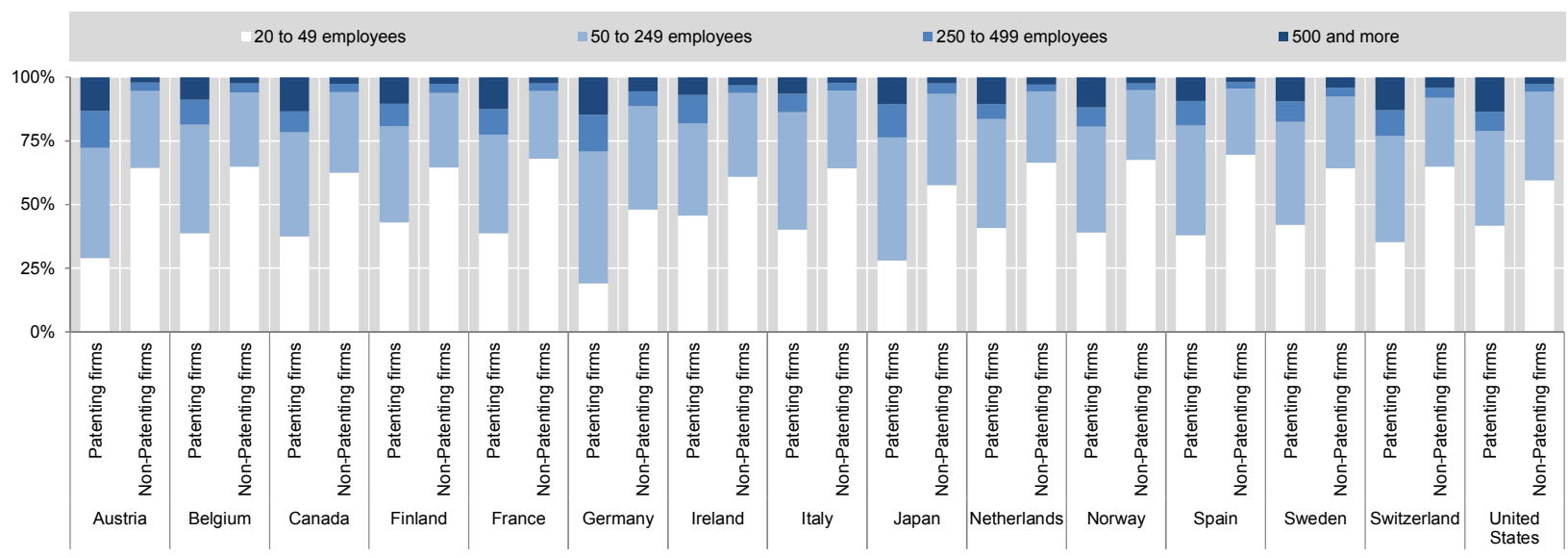

Note: Patenting firms are firms having filed for at least one patent application to the EPO, USPTO or through the PCT since 1978.

Source: OECD calculations based on EPO Worldwide Patent Statistical Database (April 2012) and OECD-ORBIS 2011, October 2012.

As Figure 2 clearly highlights, SMEs (of up to 249 employees) generally account for $75 \%$ or more of the patenting firms. The only exceptions are represented by Austria and Germany, for which this share is slightly lower. However, whereas the majority of non-patenting firms is represented by SMEs, only a very small proportion of big firms - varying between $4 \%$ and $11 \%$ - does not rely on such IP rights.

The proportion of patenting and non patenting firms by firm class size, as determined on the basis of the number of employees, is further detailed in Figure 3, which shows the share of patenting firms by firm class, in all countries considered. Whereas in terms of overall numbers SMEs seems to account for the vast majority of patent applicants, in fact the relative proportion of small and medium enterprises applying for at least one patent over the period considered remains very low. Percentages range between $1 \%$ (Spain and Canada) and $4.9 \%$ (Finland) in the case of firms of 20-49 employees, and between $1.8 \%$ (Ireland) and $12.5 \%$ (Switzerland) in the case of medium firms.

Figure 3. Share of patenting firms in total firms, by employee size class (2009)

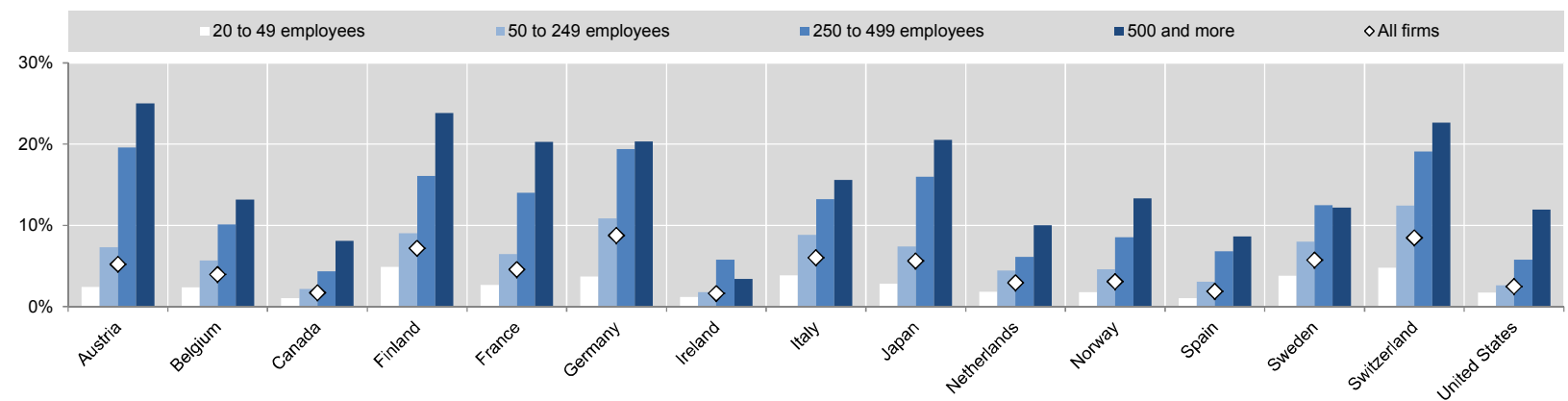

Note: Patenting firms are firms having filed for at least one patent application to the EPO, USPTO or through the PCT since 1978.

Source: OECD calculations based on EPO Worldwide Patent Statistical Database (April 2012) and OECD-ORBIS 2011, October 2012.

Figure $4 \mathrm{a}$ and Figure $4 \mathrm{~b}$ show the proportion of patent applications (Figure $4 \mathrm{a}$ ) and patent families (Figure 4b) belonging to the different size classes considered. Data relate to the year 2007-2009 and statistics rely on pooled year-specific data, in order to minimise the likelihood that the statistics shown would reflect year-specific shocks or patterns. 
Figure 4a and Figure 4b suggest that the majority of patent applications and families are generated by big firms, with the exception of Ireland and Spain ( $48 \%$ and $50 \%$ respectively) in the case of patent counts, and of Spain and Italy ( $44 \%$ and $48 \%$ respectively) in the case of patent families. Moreover, with the exception of Ireland and Canada, a general pattern emerges: the proportion of patent families applied for by SMEs consistently exceeds the proportion of patent applications filed by small and medium enterprises. These results appear in line with previous studies, and seem to suggest that smaller firms are generally more inclined than big firms to protect their intellectual property in more than one country, but that the size of their patent families is on average smaller than that of bigger firms.

Figure 4a. Distribution of patent applications by firm size classes, 2007-09, patent counts

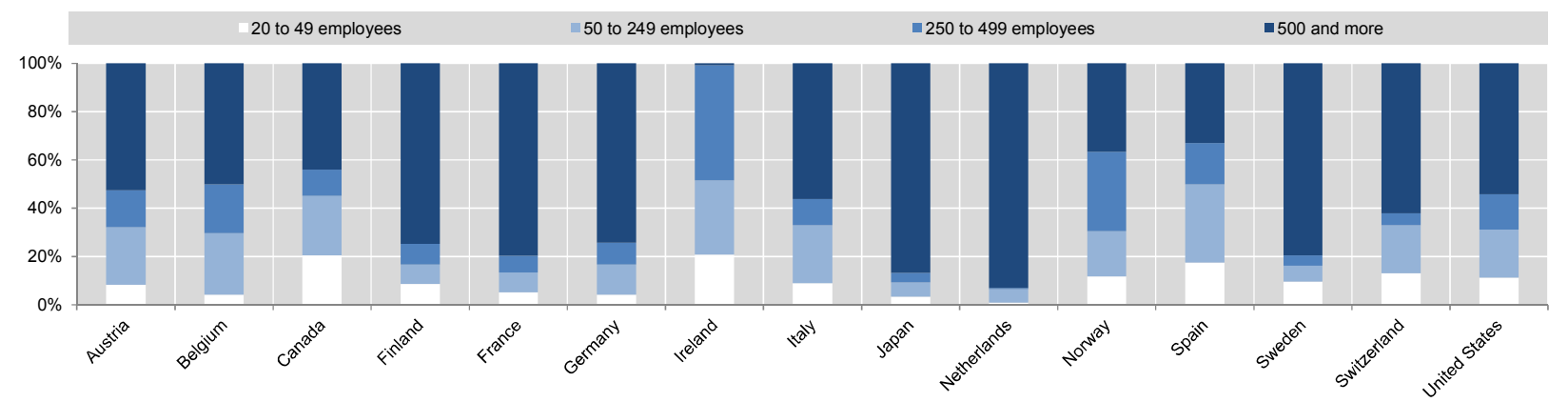

Figure 4b. Distribution of patent applications by firm size classes, 2007-09, family counts

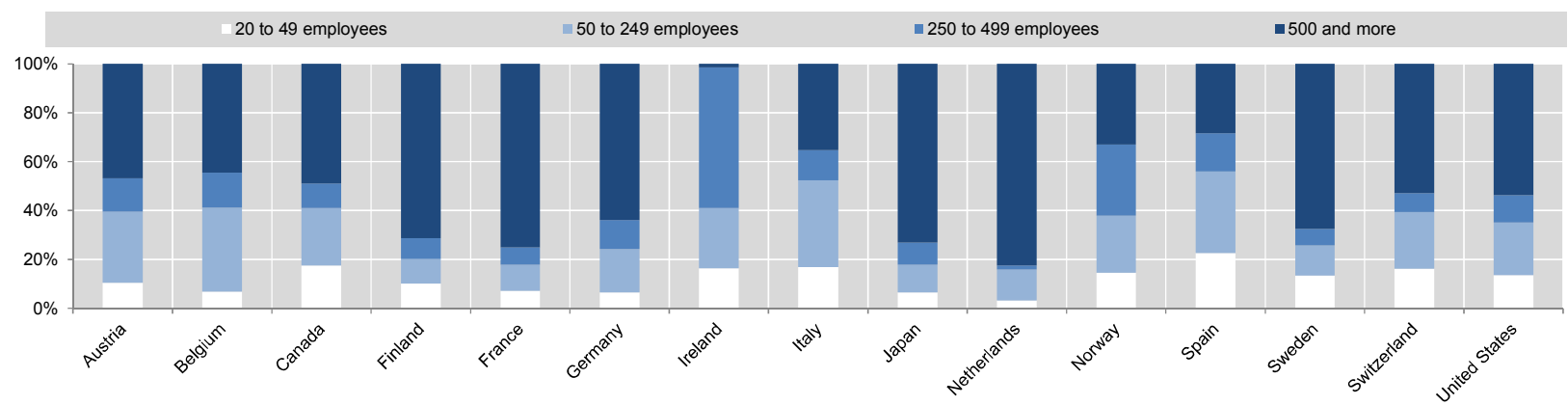

Note: Patent counts represent the number of patents filed to the EPO, the USPTO and through the PCT over the period considered, whereas the families counts controls for multiple filings of a same patent in different patent offices.

Source: OECD calculations based on EPO Worldwide Patent Statistical Database (April 2012) and OECD-ORBIS 2011, October 2012.

\section{Patenting activity by ownership status}

The following three figures aim to provide an idea of the way patenting activities by ownership status may look. They are shown here for illustrative purposes only, as the consolidation-related information contained in ORBIS $\subset$ is incomplete, and does not allow to be sufficiently confident about the ownership of firms.

Figure 5 shows the proportion of patenting firms that appear to belong to a group, according to the ownership structure information provided in ORBISC for the year 2009. It further shows the proportion of independent firms applying for patents. 
Figure 5. Distribution of patenting firms in total firms, by ownership status, 2009

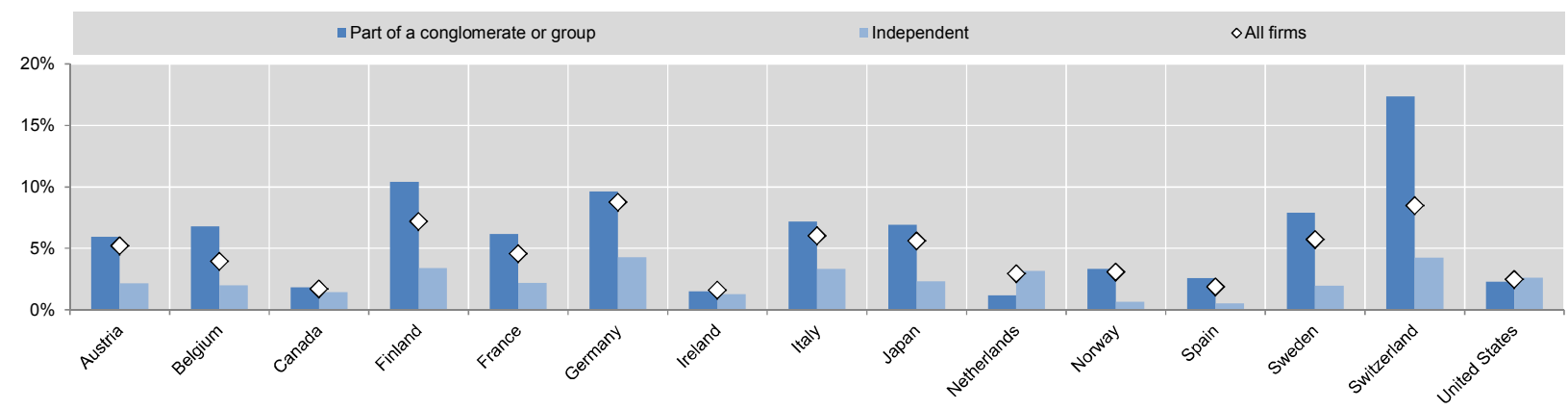

Source: OECD calculations based on EPO Worldwide Patent Statistical Database (April 2012) and OECD-ORBIS 2011, October 2012.

Figure 5 suggests that firms that belong to groups may be keener on patenting than their independent counterparts, with the exception of the Netherlands and the United States, where the number of independent firms that patent is relatively higher.

Figure $6 \mathrm{a}$ and Figure $6 \mathrm{~b}$ further show the share of patent applications that are generated by enterprises that belong to groups of conglomerates (Figure 6a) and by independent firms (Figure 6b). In both figures and for each of the countries considered, the left hand bar refers to the share of patent counts, whereas the right hand bar mirrors the percentage of patent families belonging to firms that are part of groups (Figure 6a) and to independent firms (Figure 6b).

Figure 6a. Distribution of patent applications, by ownership structure (2007-09)

Firms that are part of a conglomerate or group

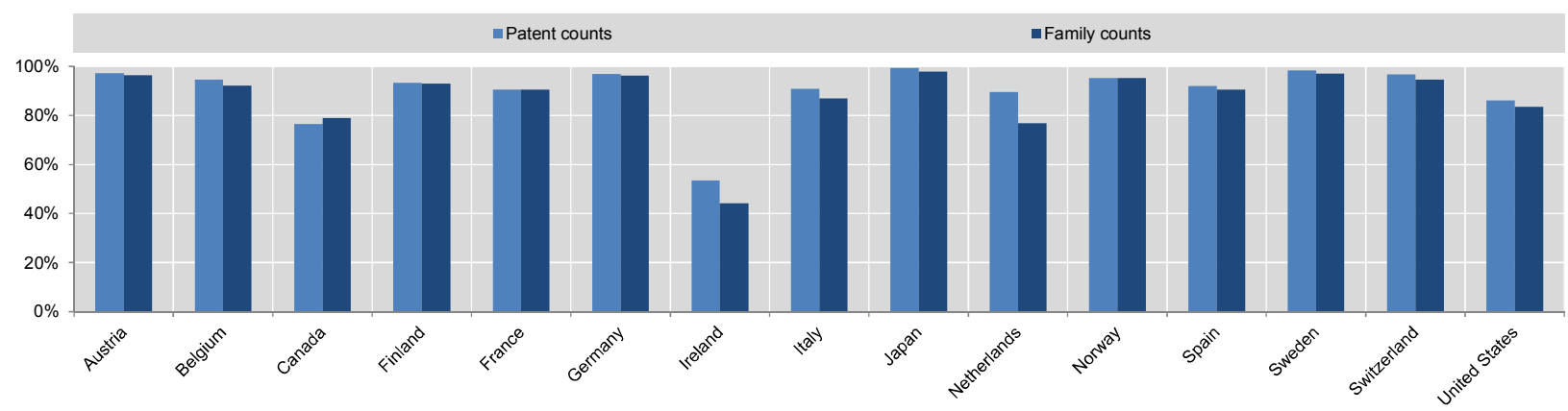

Figure 6b. Distribution of patent applications, by ownership structure (2007-09) Independent firms

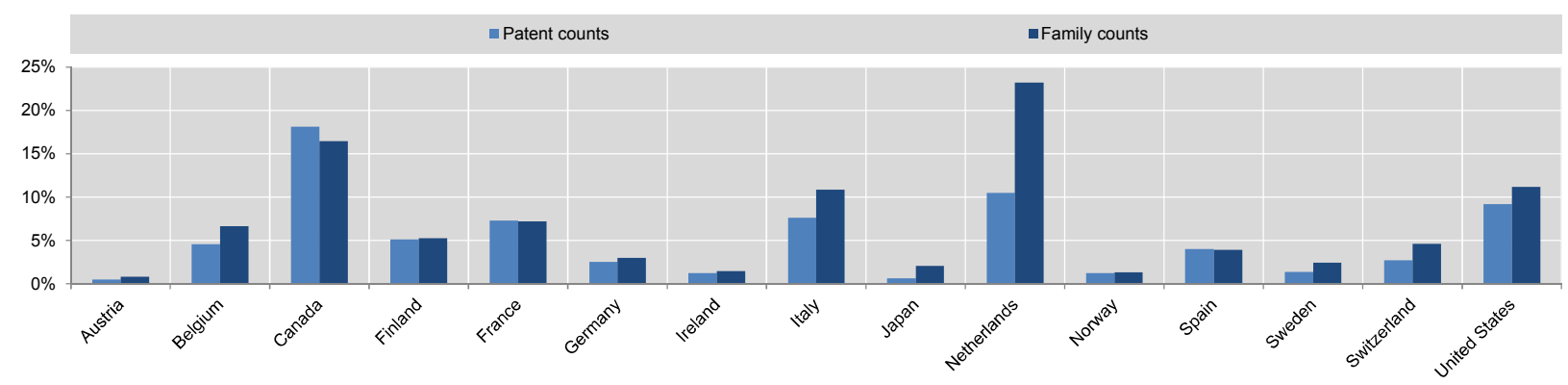

Source: OECD calculations based on EPO Worldwide Patent Statistical Database (April 2012) and OECD-ORBIS 2011, October 2012. 
In the case of firms that belong to a group or a conglomerate, the proportion of patent counts in their portfolio is typically higher - although generally slightly so - than the proportion of patent families owned by such firms. The reverse is mostly true with respect to the proportion of patent counts and patent families in the portfolio of independent firms. Canada, France and Spain represent an exception to this rule, although differences are almost negligible.

\section{Firm age and patenting activity}

This section presents a number of statistics aimed at characterising the way firms of different age classes behave, and the extent to which they patent. To this end, the age of firms has been defined as the difference between the date of patent application at EPO, USPTO or via PCT and the date of incorporation reported in OECD-ORBIS 2011. Figure 7 shows the distribution of firm age at the time of the first patent filing. Each line in Figure 7 represents one of the countries considered in the analysis and the percentages shown correspond to the proportion of firms of a certain age class that patent for the first time. For each country (i.e. line) considered, the sum of the shares observed at each of the points in time detailed in the figure adds up to $100 \%$, i.e. the total number of firms applying for the first time for a patent.

Figure 7. Age of patenting firms at the time of the first filing

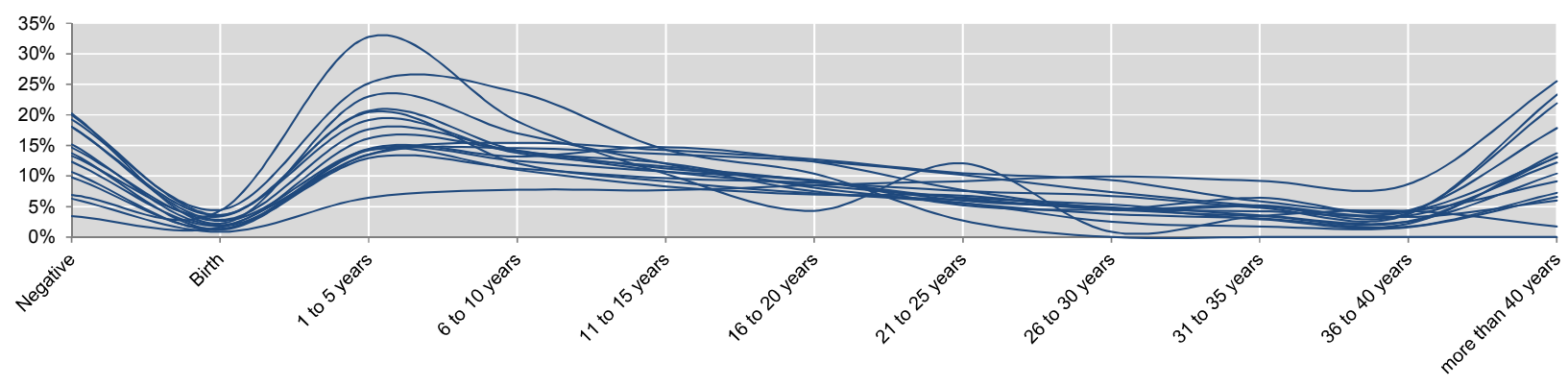

Note: The lines represent countries in the sample. The age of the patenting firm refers to the difference between the earliest date of application at EPO, USPTO or via PCT and the date of incorporation as reported in OECD-ORBIS 2011.

Source: OECD calculations based on EPO Worldwide Patent Statistical Database (April 2012) and OECD-ORBIS 2011, October 2012.

Some common patterns emerge from the analysis of Figure 7. In all countries considered, the majority of first patenting events happens between the birth of a firm and its tenth year of age. The likelihood to patent for the first time decreases gradually after that year, and seems to grow again in the case of very old firms, i.e. of firms of 40 or more years of age. This last pattern may be due to the way incorporation dates are recorded in ORBIS $\odot$, e.g. in the case of spin-offs or newly established affiliates being imputed the incorporation date of the "mother" company. A notable proportion of firms further seems to apply for patents even before being established (firms here denoted as being of "negative age"). This may be the case when start-ups are created in order to exploit the innovative property previously developed by its founders; or when mergers and acquisitions regard firms having patents in portfolios that pre-date the creation of the merging or acquiring firm. Simple errors related to the imputation of the date of incorporation of firms may further explain the statistics observed at the tails of the distributions.

Table 8 further investigates the "negative age" issue by looking at the overall number of firms that seemingly patent before being established (showed in the right hand column of Table 8), and by subdividing firms according to the time lag that elapses between their first filing for a patent and the date in which they get incorporated. 
Table 8. Patenting firms with negative age at first filing

\begin{tabular}{lccc|c}
\hline & $\begin{array}{c}\text { up to } \mathbf{4} \text { years } \\
\text { before }\end{array}$ & $\begin{array}{c}\mathbf{5} \text { to } \mathbf{1 0} \text { years } \\
\text { before }\end{array}$ & $\begin{array}{c}\text { more than 10 } \\
\text { years before }\end{array}$ & $\begin{array}{c}\text { Patenting firms } \\
\text { reporting negative age }\end{array}$ \\
\hline Austria & $43 \%$ & $34 \%$ & $22 \%$ & 145 \\
Belgium & $34 \%$ & $30 \%$ & $36 \%$ & 76 \\
Canada & $42 \%$ & $27 \%$ & $31 \%$ & 475 \\
Finland & $38 \%$ & $37 \%$ & $25 \%$ & 138 \\
France & $36 \%$ & $21 \%$ & $44 \%$ & 656 \\
Germany & $47 \%$ & $24 \%$ & $29 \%$ & 1673 \\
Ireland & $50 \%$ & $50 \%$ & $\ldots$ & 8 \\
Italy & $37 \%$ & $30 \%$ & $33 \%$ & 590 \\
Japan & $37 \%$ & $27 \%$ & $35 \%$ & 324 \\
Netherlands & $51 \%$ & $26 \%$ & $23 \%$ & 122 \\
Norway & $38 \%$ & $27 \%$ & $35 \%$ & 78 \\
Spain & $67 \%$ & $20 \%$ & $14 \%$ & 51 \\
Sweden & $37 \%$ & $27 \%$ & $36 \%$ & 148 \\
Switzerland & $44 \%$ & $25 \%$ & $30 \%$ & 311 \\
United States & $37 \%$ & $24 \%$ & $39 \%$ & 5122 \\
\hline
\end{tabular}

Source: OECD calculations based on EPO Worldwide Patent Statistical Database (April 2012) and OECD-ORBIS 2011, October 2012.

Simple country averages suggests that in $43 \%$ of cases, firms first file for a patent up to 5 years before being established. These filings are likely related to firms that are later established in order to exploit a certain innovative portfolio. $29 \%$ and $31 \%$ of firms instead appear to file for the first time for a patent between 5-10 years and more than 10 years before being established, respectively. These latter firms are likely to have been interested by the structural changes mentioned above, although such considerations remain merely speculative in the absence of additional pieces of information.

Figure 8 offers a snapshot for the period 2005-2007 of the age of firms at the time of their first filing. The left hand bars refer to the proportion of young firms, of up to 5 years of age, that apply for the first time for a patent during the years considered. The right hand bars instead show the percentage of firms that apply for the first time for a patent, regardless of their age. In countries like Canada, Ireland, Japan and the United States young firms seem to have a higher likelihood to patent than the rest of the firms. The reverse seems to be true in a number of other countries, including Germany, Italy and Sweden.

Figure 8. Firms having first filed for a patent in $2005-07$, by age at date of first filing, in total firms of same age

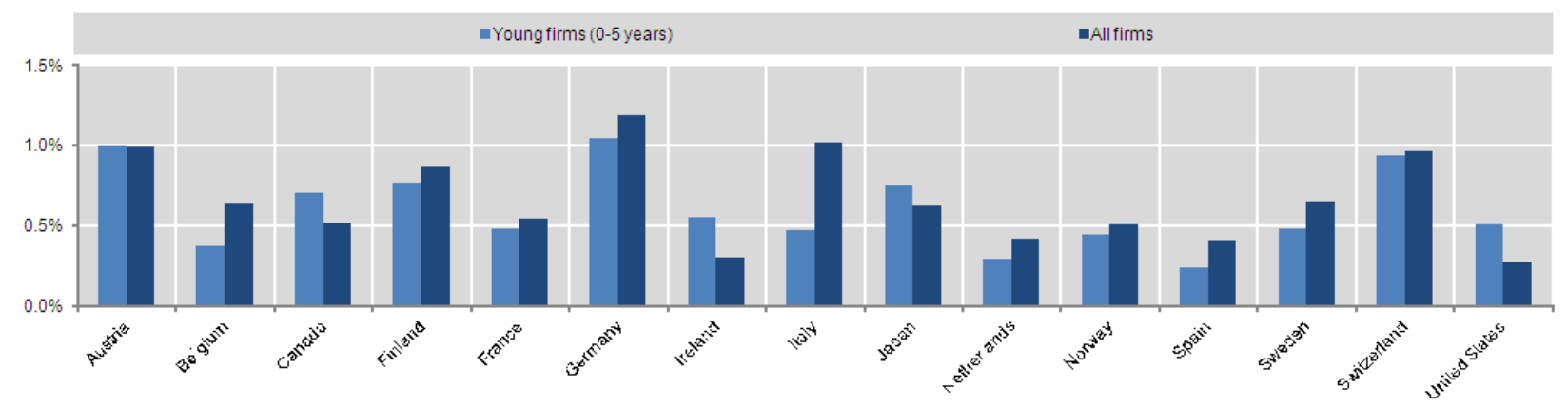

Note: The age of the patenting firm refers to the difference between the earliest date of application at EPO, USPTO or via PCT and the date of incorporation as reported in OECD-ORBIS 2011.

Source: OECD calculations based on EPO Worldwide Patent Statistical Database (April 2012) and OECD-ORBIS 2011, October 2012. 


\section{Patenting activity at the sectoral level}

This section presents a number of descriptive statistics aiming at characterising the sector-specific patenting behaviours of firms. Figure 9 shows the distribution of patenting activities across sectors, defined following the ISIC Rev. 4 classification (corresponding to the NACE Rev. 2 classification). The list of sectors included in the analysis can be seen in Table 1A, in the Annex.

Figure 9.a refers to all patenting firms, throughout the twelve year period considered. Figure 9.b shows some statistics related to firms filing patent applications during the years 2005-2009. Finally Figure 9.c shows the sector-specific proportion of firms that do not rely on this type of intellectual property rights. Each figure highlights the range of values observed in the fifteen countries considered, i.e. the minimum and maximum values observed for each sector, as well as the resulting average values. The latter have been constructed over the pooled data and therefore correspond to weighted averages, with weights that are determined by the overall number of country and sector-specific observations available.

Figure 9. Distribution of firms, by industry classes (ISIC, rev.4)

a. All patenting firms

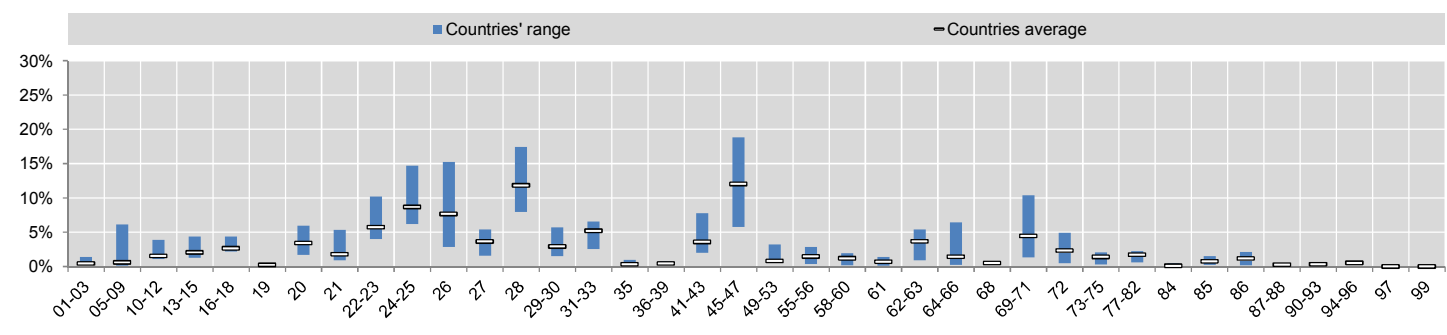

b. Patenting firms with patent applications in 2005-09

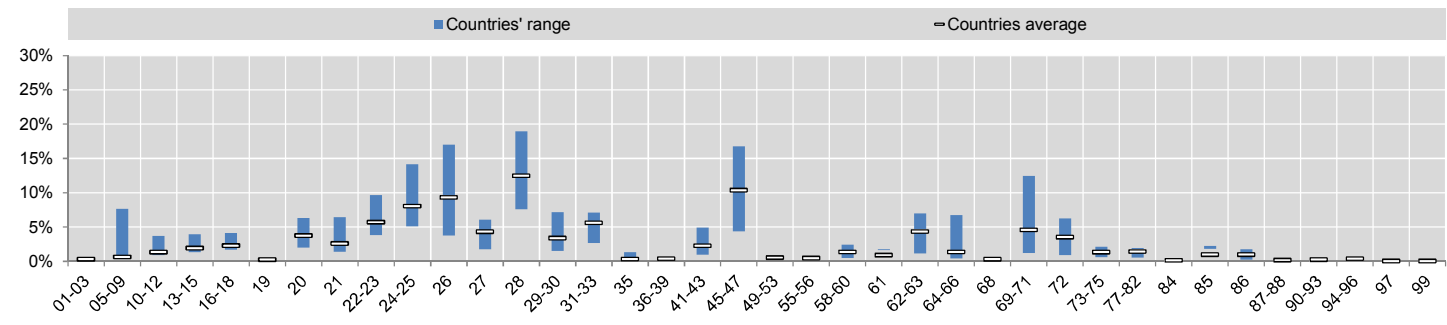

c. Non patenting firms

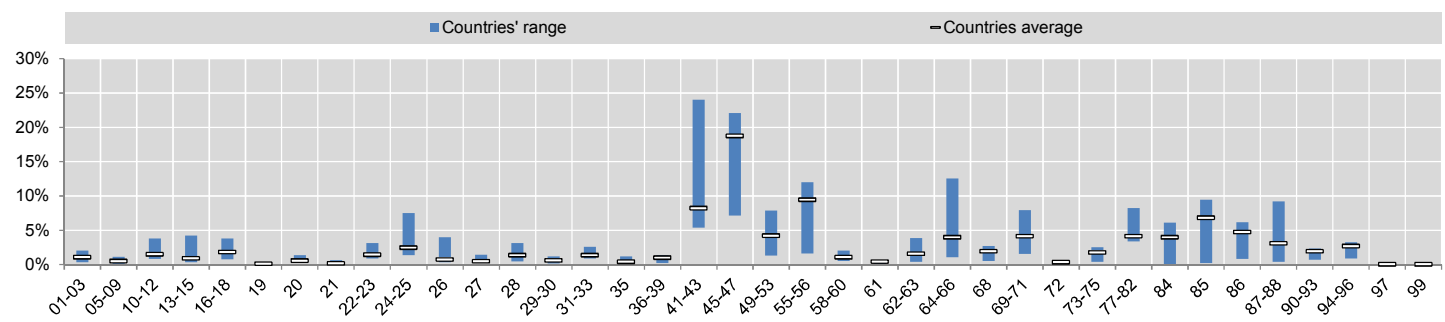

Note: Sector-specific ranges are determined by the minimum and the maximum shares observed in any of the countries considered.

Source: OECD calculations based on EPO Worldwide Patent Statistical Database (April 2012) and OECD-ORBIS 2011, October 2012.

The same statistics are proposed for the overall 12-year period considered, as well as for a 5 -year subset (2005-2009), in order to give a flavour of the sensitivity of figures to the length of the time period considered, and to the sample selection that a shorter window of observations might imply. As can be seen, 
the shares shown in Figure 9 do not appear to vary much when different time periods are considered. The sectors that seem to consistently account for a relatively high share of patent applications (of around $10 \%$ or more) are: sector 26 "Computer, electronic and optical products"; sector 28 "Machinery and equipment n.e.c."; and sectors 45-47 "Wholesale and retail trade, repair of motor vehicles and motorcycles". Sectors 45-47 are also the sectors featuring on average the higher share of non patenting firms, as can be seen from Figure 9.c.

Figure 10. Proportion of patenting firms in total, by industry (ISIC, rev.4) and size class
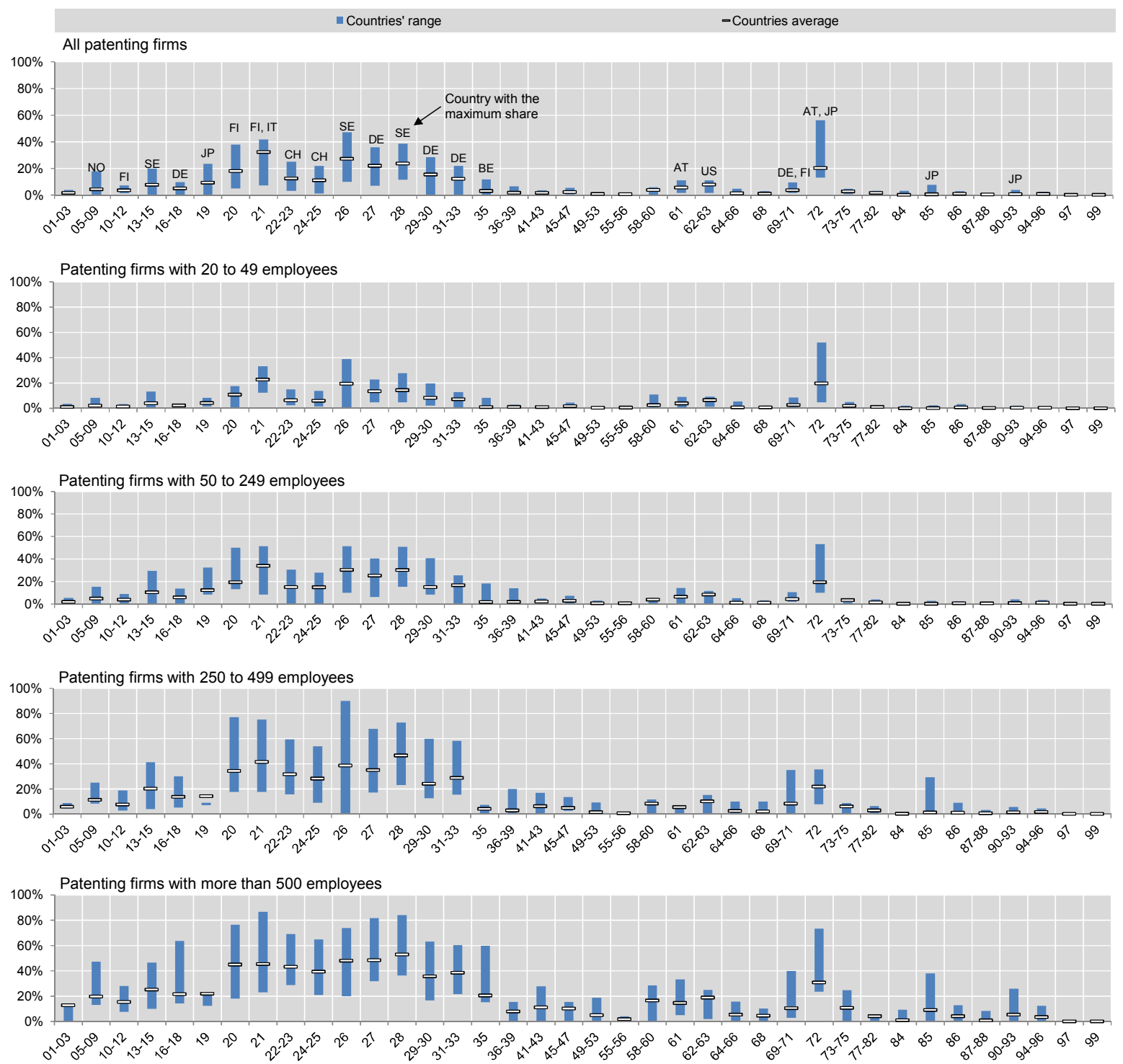

Note: Firms having filed for at least one patent application at EPO, USPTO or through the PCT since 1978. Sector-specific ranges are determined by the minimum and the maximum shares observed in any of the countries considered.

Source: OECD calculations based on EPO Worldwide Patent Statistical Database (April 2012) and OECD-ORBIS 2011, October 2012.

Figure 10 provides a wealth of information related to the way patenting activities are distributed across sectors and size classes. It further points out the countries that exhibit the highest sector-specific share of patenting firms. When proposing statistics by size class, Figure 10 also denotes the minimum and maximum proportion of patenting firms in the sectors considered. 
The highest average shares of patenting firms can be observed in sectors 20 to 35 (i.e. from chemicals and pharmaceuticals to transport, furniture and electricity) and in sector 72 ("Scientific Research and Development") across all size classes considered. This is consistent with previous studies and with what is known about the innovative activity of these sectors.

Figure 11 offers the same statistics proposed in Figure 10, but focuses on the period 2005-2009. This is done to give an indication of the sensitivity of results to the specific time frame considered. A comparison of Figure 10 with Figure 11 highlights the consistency of the statistics shown, with the sectoral patterns emerging from the two sets of statistics compiled that appear very similar. Notable differences can be observed with respect to the countries featuring the highest share of patenting firms by sector.

Figure 11. Proportion of patenting firms in total, by industry (ISIC, rev.4) and size class - employees
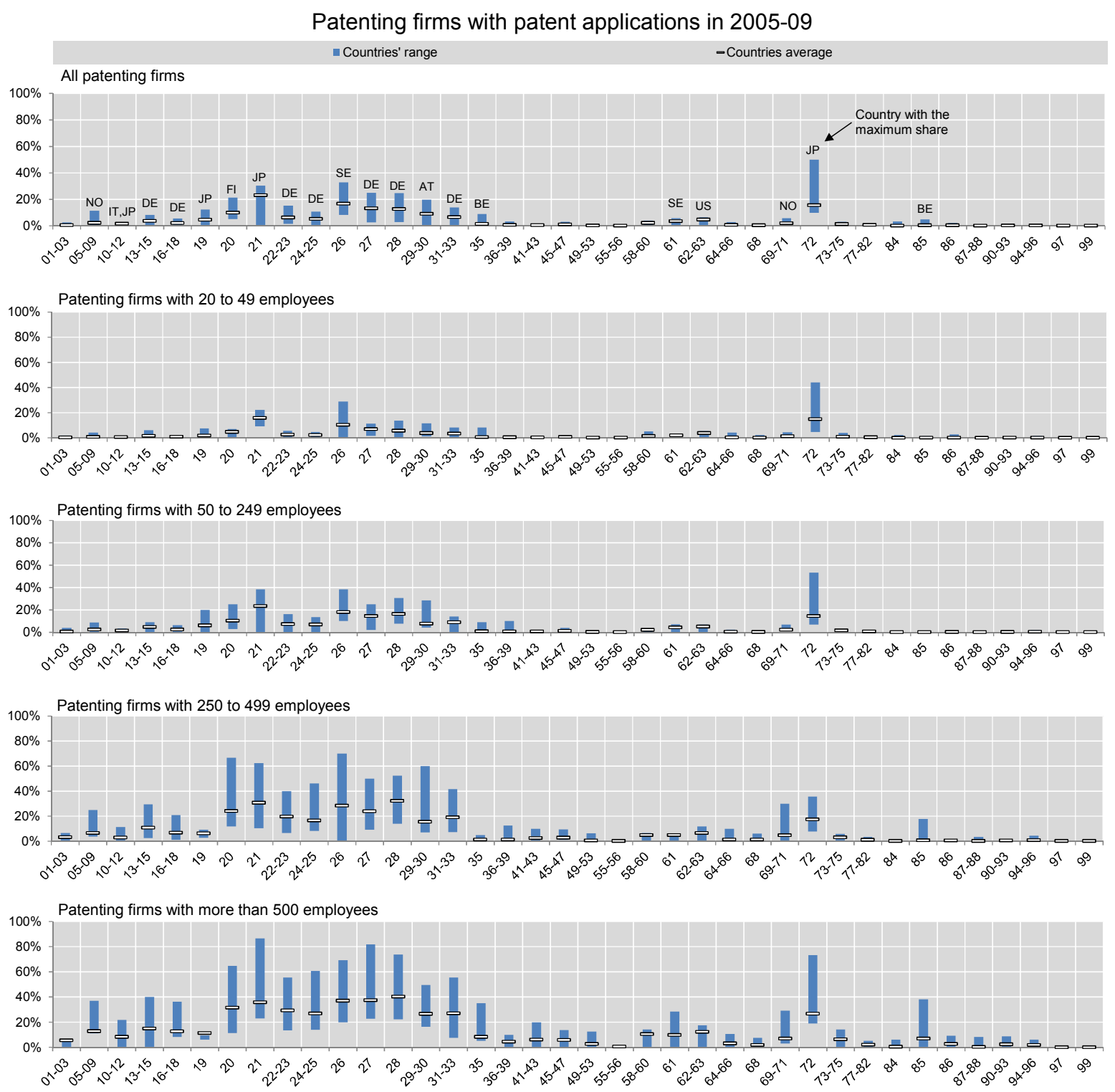

Note: Firms having filed for at least one patent application at EPO, USPTO or through the PCT between 2005 and 2009 . Sectorspecific ranges are determined by the minimum and the maximum shares observed in any of the countries considered.

Source: OECD calculations based on EPO Worldwide Patent Statistical Database (April 2012) and OECD-ORBIS 2011, October 2012. 
Figure 12 shows the distribution of patent filings by size class and by industry and highlights the countries showing the highest share of patent filings in the sectors considered. Figure 13 additionally shows the same statistics of Figure 12 while accounting for patent families. Average data refer to the proportion of patents belonging to the sector considered, out of the total number of patents originating from the size classes detailed. It should further be noted that in some cases high percentages may be due to the low number of observations.

Figure 12. Distribution of patent filings, by size class and by industry (ISIC, rev.4), 2005-09
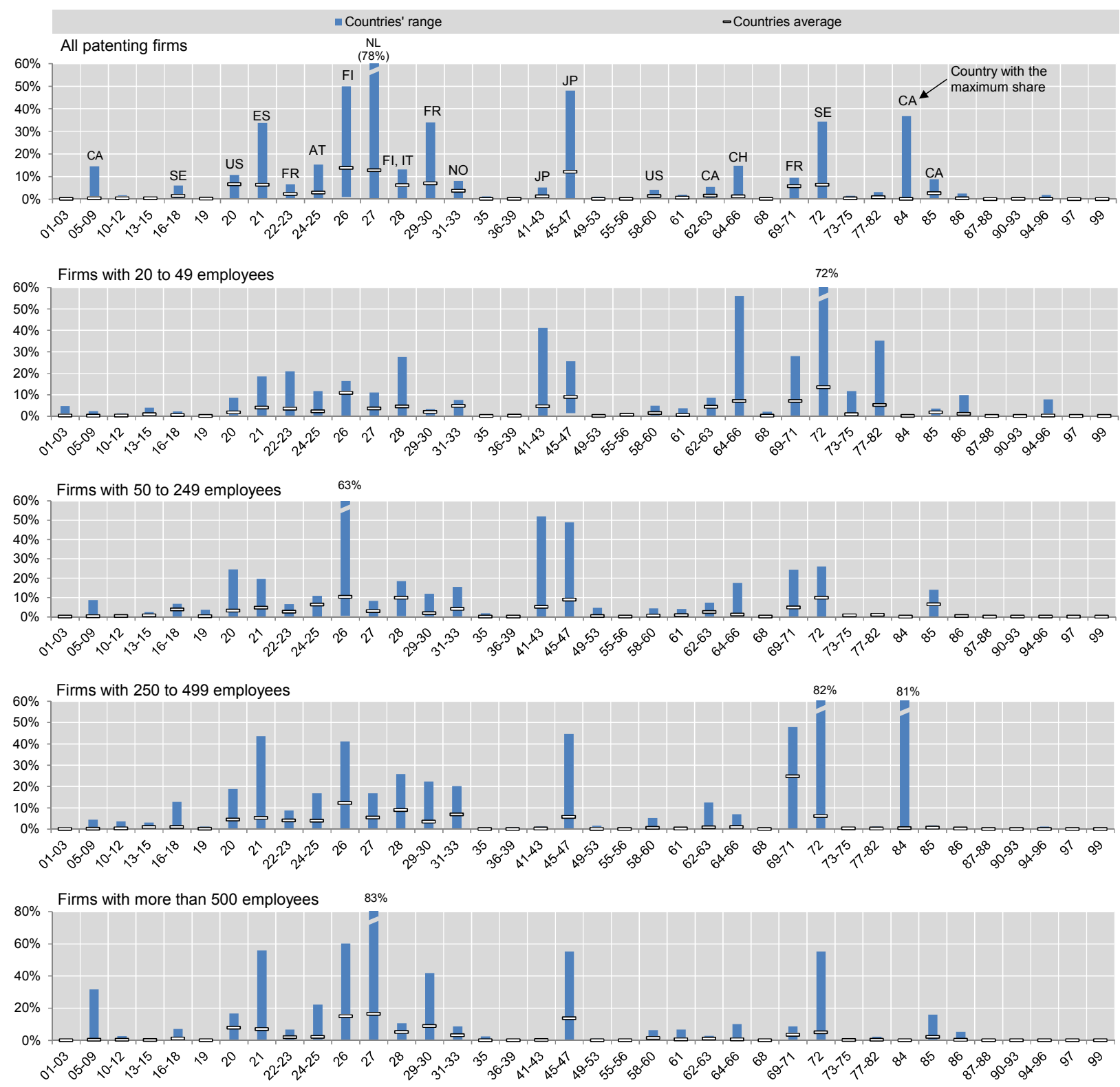

Note: Patent applications filed to EPO, USPTO or through the PCT between 2005 and 2009. Sector-specific ranges are determined by the minimum and the maximum shares observed in any of the countries considered.

Source: OECD calculations based on EPO Worldwide Patent Statistical Database (April 2012) and OECD-ORBIS 2011, October 2012. 
Figure 13. Distribution of patent filings (families adjusted), by size class and by industry (ISIC, rev.4), 2005-09
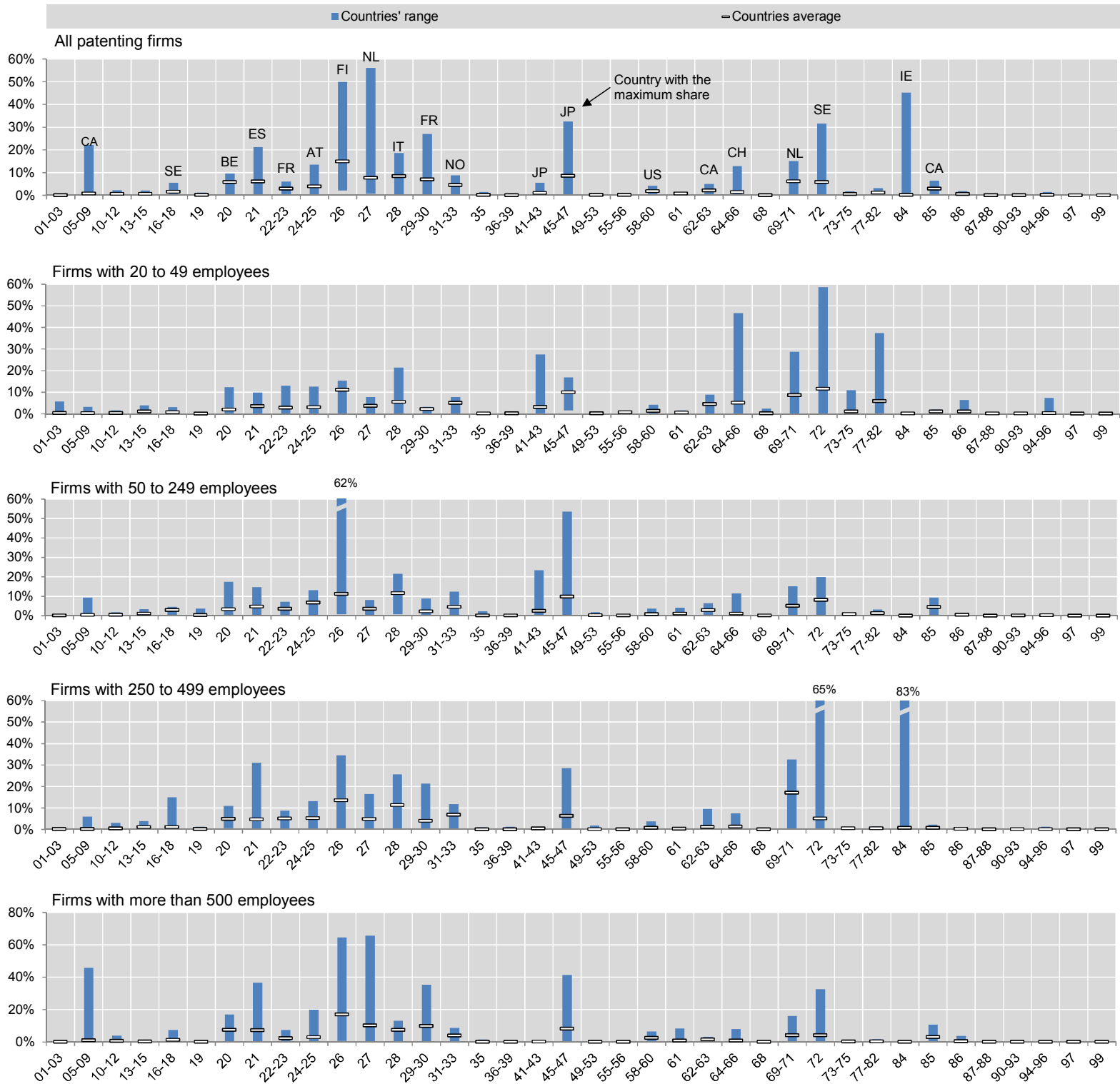

Note: Families of patent applications filed to EPO, USPTO or through the PCT between 2005 and 2009. Sector-specific ranges are determined by the minimum and the maximum shares observed in any of the countries considered.

Source: OECD calculations based on EPO Worldwide Patent Statistical Database (April 2012) and OECD-ORBIS 2011, October 2012.

Results appear consistent with what is known about the sectoral innovative activity of firms in different countries. Examples are the Netherlands in sector 27 "Electrical equipments", Finland in sector 26 "Computer, electronic and optical equipment", and France in sector 29-30 "Transport equipment". Moreover, the countries contributing the largest sector-specific shares of patents only seldom change when patent families are accounted for. Finally, industry structure seems to indeed matter, as the proportion of innovators in some sectors is very high only in certain firm size classes. Examples are sectors 41-43 "Construction" with patents that are mostly filed by small firms, and sector 84 "Public administration and defence, compulsory social security" where almost only firms between 250 and 499 employees appear to seek patent protection. 


\section{Patent renewals by sector}

The renewal of a patent by a firm signals that the knowledge and R\&D output described in the patent document is still useful for the firm, as no rational agent would be willing to pay money for a right that is of no value. This explains why patent renewal models use the information on patent renewals to estimate the value of patents, in analyses where firms maximise the present discounted value of their returns to R\&D investment (e.g. Pakes and Schankerman, 1984). Such models rely on evidence showing that patents are highly correlated to $R \& D$ expenditures, and that changes in $R \& D$ expenditures are typically paralleled by changes in patenting behaviours (Griliches, 1998). Moreover, R\&D appears to be contemporaneous to patenting, and possible lag effects are small and not well estimated (Hall, Griliches, and Hausman 1986).

Here the focus is on the extent to which patents are renewed across sectors, to shed some light on the length of time for which such IPR prove useful to innovators. Statistics rely on a revealed preference argument, as they build on all explicit signals that firms send about the usefulness or not of their intellectual property assets. These correspond to annual renewals of patents granted, as well as to the withdrawals of patents. In the case of patent renewals, the life of a patent corresponds to the latest year for which patent renewal fees have been paid. For example, a patent applied for in 1999, granted in 2003 and renewed until 2005 will be considered as having had a life of 6 years. In the case of withdrawn patents, patent life length has been calculated as the difference between the year of application and the year of withdrawal. For example, a patent filed in 1999 and withdrawn in 2002 will have a life length of 3 years.

The statistics shown in the present document rely on data from the EPO's Worldwide Legal Status (also known as INPADOC Legal Status database) and consider the following pieces of information:

- PGFP: Annual fees paid to the national office;

- 18D: Patent deemed withdrawn;

- 18W: Patent withdrawn;

- PG25: Lapsed in a contracting state announced via postgrant information from national office to EPO.

Figure 14 shows the share of patent filed between 1978 and 1991 according to the length of time they have been renewed for, i.e. 1 to 5 years, 6-10 years, 11-15 years and 16-20 years. The complement to $100 \%$ is given by those patent applications that were not granted IPR protection and those for which the legal status is unknown. Only renewals of patents filed until 1991 are accounted for, as otherwise it would not be possible to observe the latest possible renewal event (i.e. renewal at the $20^{\text {th }}$ year) for all patents. In other words, a 20-year window of observation period needs to be allowed after the filing date. The top part of Figure 14 shows the grant and renewal rates of the patents filed by firms in the agriculture, mining, manufacturing and constructions sectors, whereas the bottom part of Figure 14 relates to the services sectors. The small numbers at the top of each bar signal the overall number of patents filed at the sector level over the period considered. 
Figure 14. Share of EPO patents filed in 1978-91 by life duration (years) and by sector

a. Agriculture, mining, manufacturing and construction

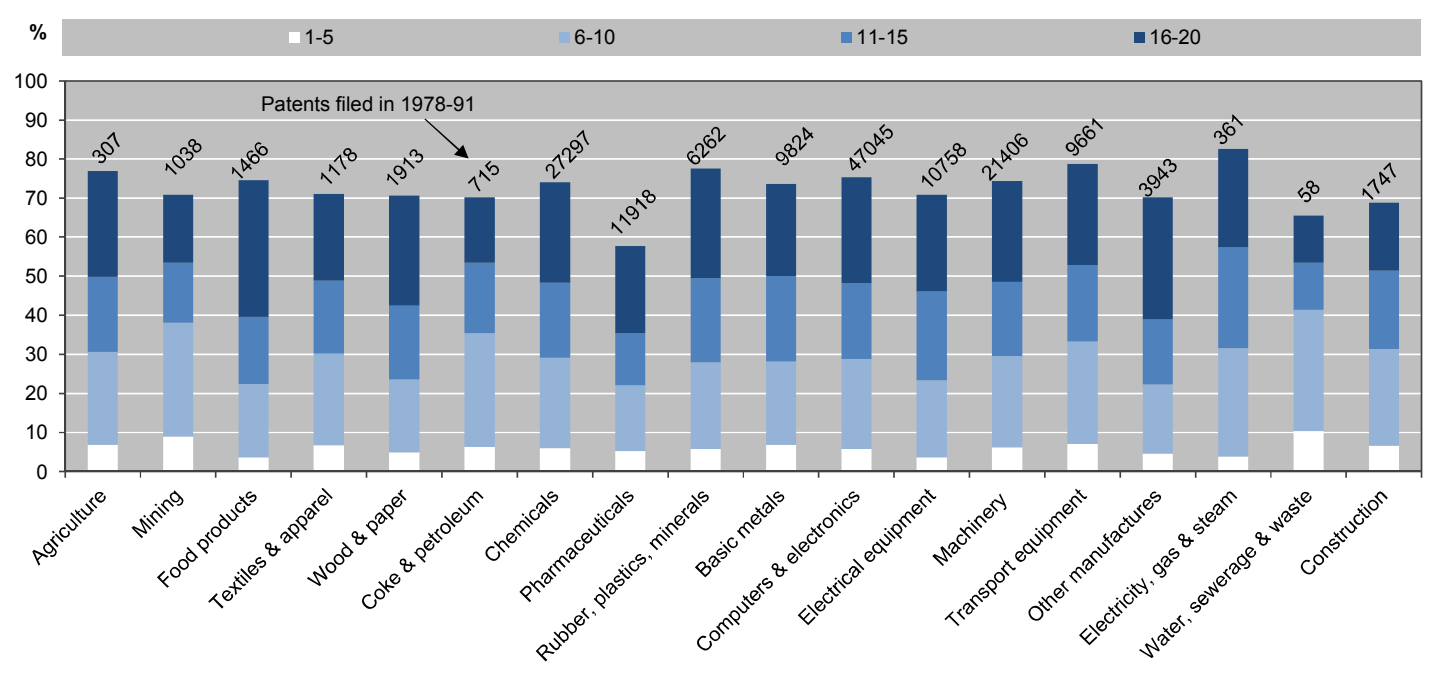

b. Services sectors

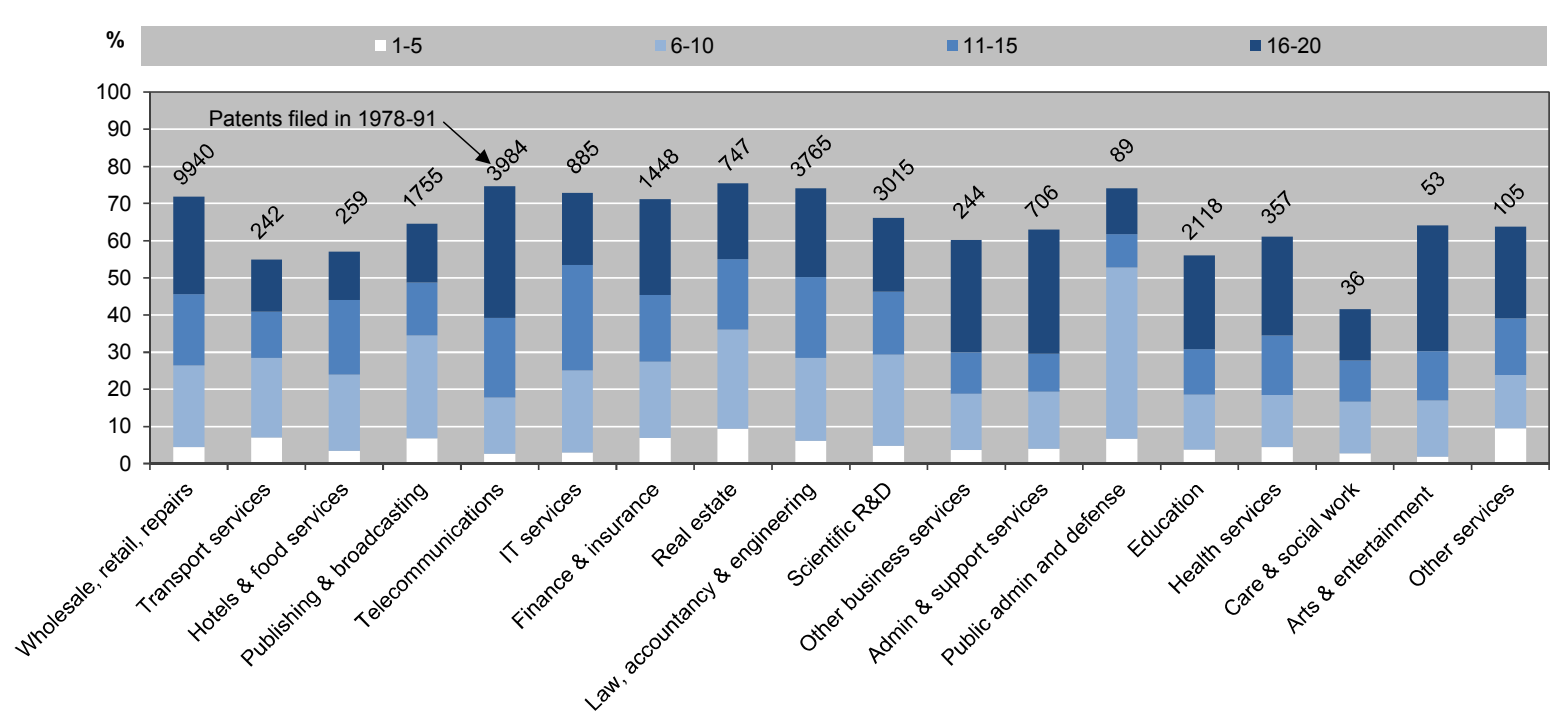

Note: residual percentages correspond to patent applications of unknown status or that have not been granted.

Source: OECD calculations based on the Worldwide Patent Statistical database (PATSTAT) and Worldwide Legal Status database (INPADOC), EPO, April 2012; and OECD-ORBIS 2011, October 2012.

Figure 14 highlights that a proportion of granted patent applications varying between $14 \%$ and $26 \%$ gets renewed up to 16-20 years. This is true also and especially for sectors like 61 "Telecommunications", whose technological paradigm is supposed to change rapidly over time. It can be further noted that patent renewals patterns are indeed sector-specific, and may mirror the composition of the sector as well as its competition dynamics.

Figure 15 shows the life duration of granted patents and looks at patent applications filed during the period 1978-1991. Similarly to Figure 14, Figure 15 statistics are presented in two panels, one for manufacturing and the other for services. Each panel shows the sector-specific average and median 
renewal lengths, as well as the value of the $1^{\text {st }}$ and $99^{\text {th }}$ percentile, in order to give an idea of minimum and maximum values. The small numbers at the top of the bars mirror the number of observations, i.e. of granted patents, on which calculations rely.

Figure 15. Life duration of EPO patent grants, applications filed in 1978-91

a. Agriculture, mining, manufacturing and construction

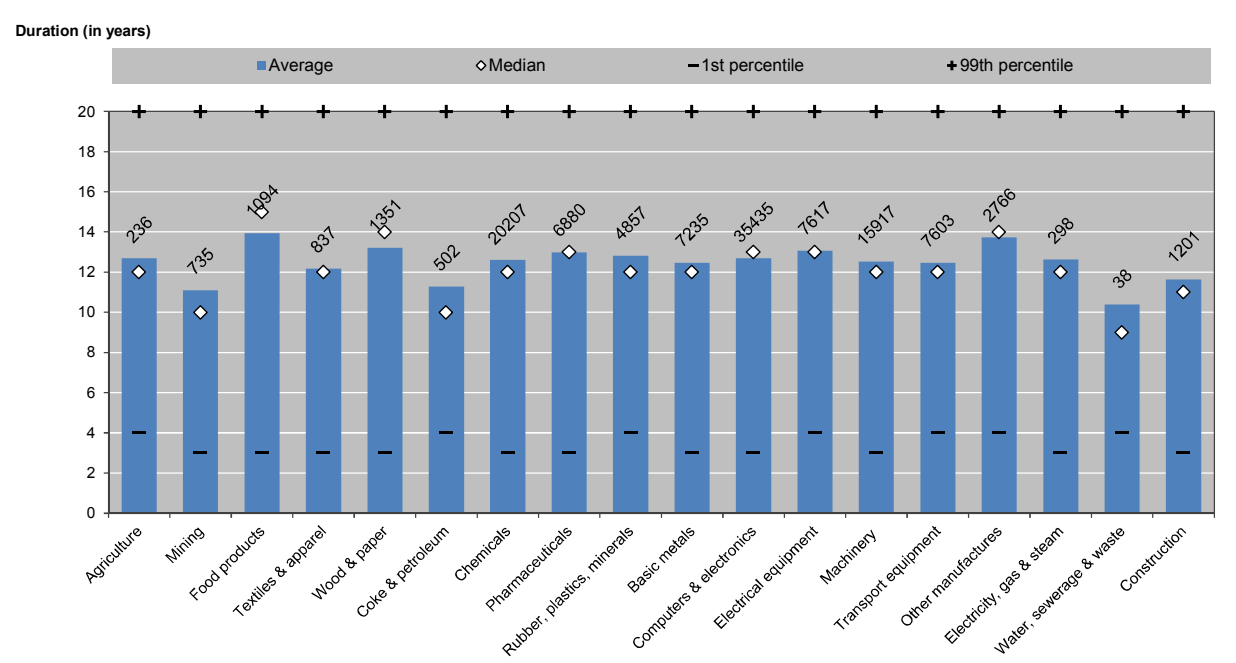

b. Services sectors

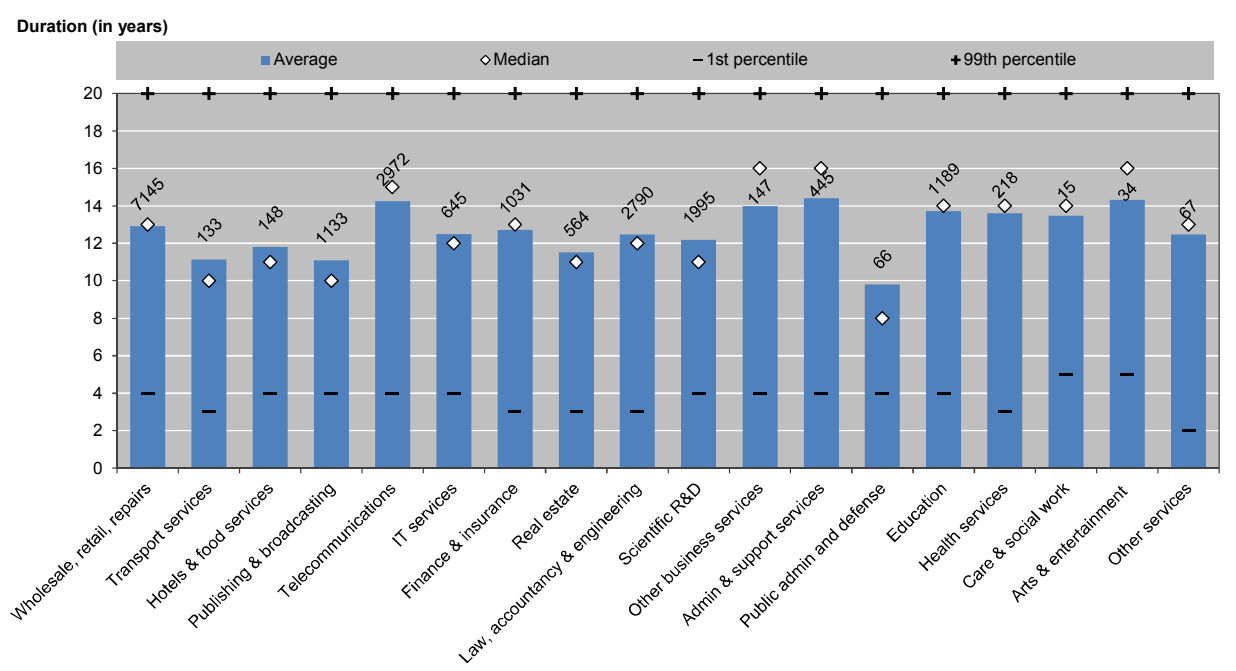

Source: OECD calculations based on the Worldwide Patent Statistical database (PATSTAT) and Worldwide Legal Status database (INPADOC), EPO, April 2012; and OECD-ORBIS 2011, October 2012.

For the whole sample, an average renewal value of 12.7 years, a median renewal value of 13 years, and $1^{\text {st }}$ and $99^{\text {th }}$ percentile values of 3 and 20 years respectively are observed. A comparison of sectorspecific average and median values suggests the existence of both right- and left-skewed sector-specific distributions, which again possibly mirror sectors' structure and dynamics.

Figure 16 illustrates the sector-specific patent renewal behaviours of small and medium enterprises, and presents a set of information similar to the one showed in Table 14. Figure 17 shows the life duration of patents granted to SMEs and regards patent applications filed during the period 1978-1991. 
Figure 16. Share of EPO patents filed in 1978-91 by duration (years) and sector - firms with 20-250 employees a. Agriculture, mining, manufacturing and construction

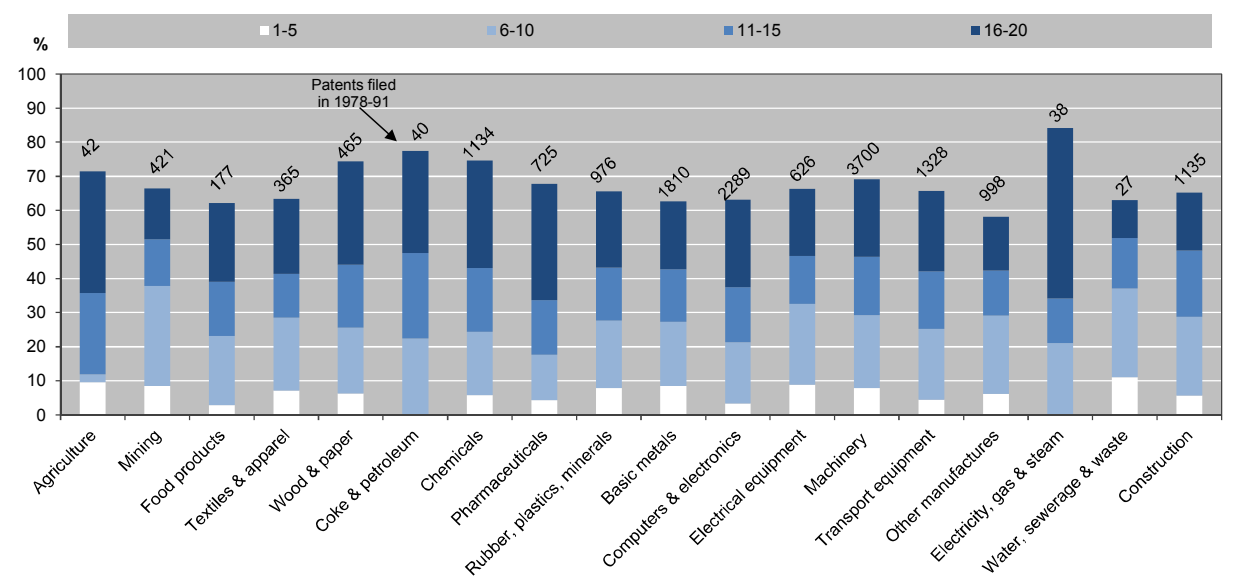

b. Services sectors

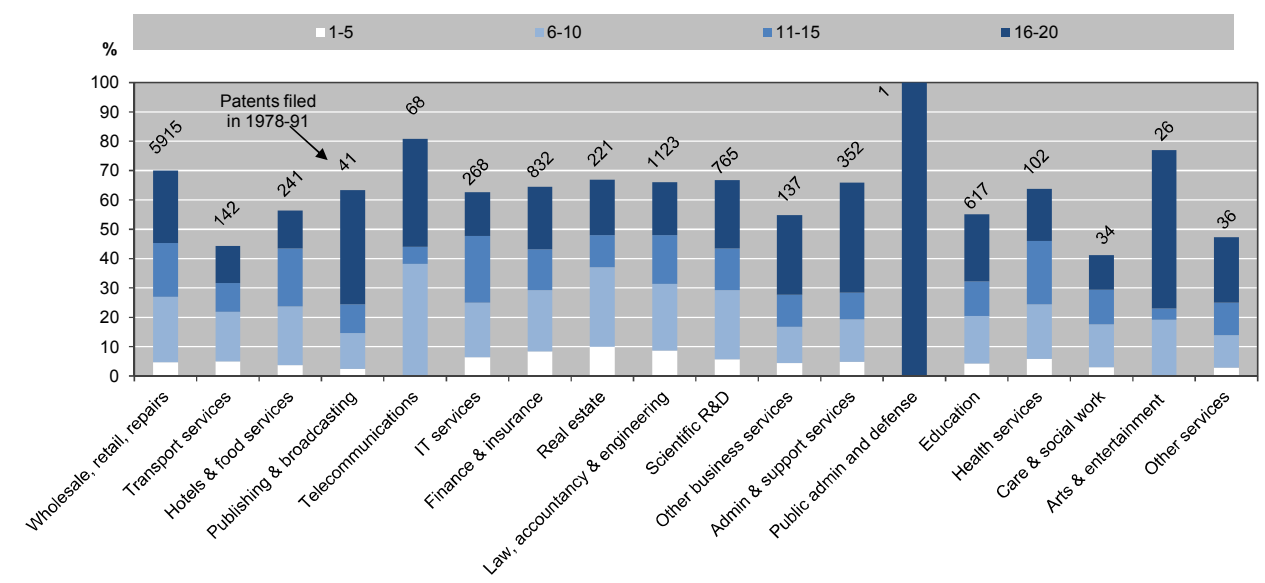

Note: residual percentages correspond to patent applications of unknown status or that have not been granted.

Source: OECD calculations based on the Worldwide Patent Statistical database (PATSTAT) and Worldwide Legal Status database (INPADOC), EPO, April 2012; and OECD-ORBIS 2011, October 2012. 
Figure 17. Life duration of EPO patent grants, applications filed in 1978-91 - firms with 20-250 employees a. Agriculture, mining, manufacturing and construction

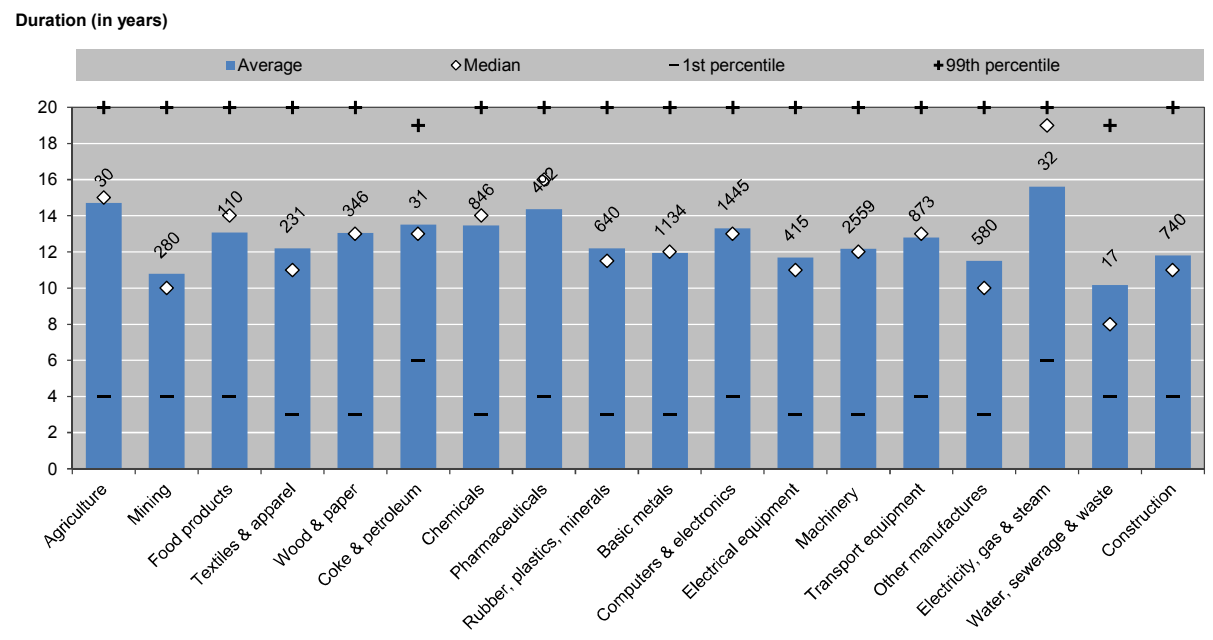

b. Services sectors

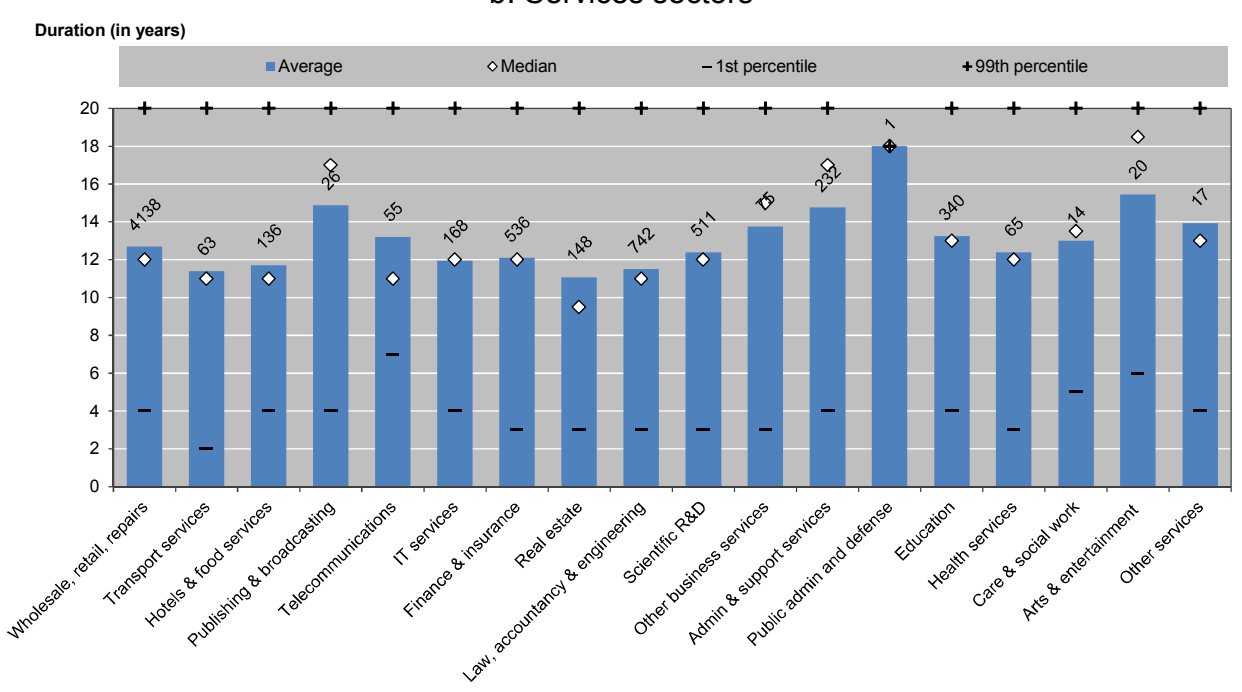

Source: OECD calculations based on the Worldwide Patent Statistical database (PATSTAT) and Worldwide Legal Status database (INPADOC), EPO, April 2012; and OECD-ORBIS 2011, October 2012

When small and medium enterprises are considered, an overall average renewal period of 12.4 years, a median renewal length of 12 years, and $1^{\text {st }}$ and $99^{\text {th }}$ percentile values of 3 and 20 years, respectively, can be observed. Hence, although sector-specific differences can seem to exist in the renewal patterns of all firms and those of SMEs, aggregate values for the full sample and the subset of SMEs do not differ by much. This possibly underlines the importance of sector-specific renewal patterns, and that sector and technology specific features influence the renewal behaviours of small and big players alike. However, the possibility that the selection of SMEs contained in the commercial database that is used may be affecting these results, cannot be dismissed.

\section{Conclusions}

A vast literature exists that deals with the use of patents by firms and with the way patenting relates to various performance indicators like firm survival and productivity. Often due to data availability 
constraints, these studies nevertheless focus on one or few technological domains, industries, or countries at a time, making it difficult to generalise results and to uncover common patterns and possible determinants across countries and over time, in support of policy making.

This study attempts to partially address such a shortcoming by proposing an exploratory characterisation of firms' patenting behaviours across fifteen countries over the period 1999-2010. It relies on patent data matched to firm data from a commercial dataset and tries to depict a broad picture about the patenting activities of firms of different size, age and industry.

The patent-related analysis is preceded by a series of statistics aimed at describing some of the main characteristics and shortcomings of the firm-level commercial dataset used. The results of this simple exercise underline the limits inherent in using commercial databases to provide robust evidence in support of policy making and the need to find ways to access business registers data for cross country analysis purposes.

The study is descriptive in nature and exploratory in aim. Future analysis will provide evidence about the 'quality' versus 'quantity' debate, i.e. the extent to which firms of different age, size and sector generate 'high quality' inventions, i.e. inventions of high technological and economic value, having an impact on subsequent technological developments. Future work will also be devoted to empirically (i.e. econometrically) investigate the relationships that may exist between firms' innovative behaviours and their performance, and to uncover the role that framework conditions might have on such patterns. 
DSTI/DOC(2013)5

\section{ANNEX}

Table 1A. International Standard Industrial Classification of all Economic Activities (ISIC), Rev.4

\begin{tabular}{|c|c|}
\hline \multicolumn{2}{|c|}{ Industry list (ISIC, rev.4) } \\
\hline $01-03$ & Agriculture, Hunting, Forestry and Fishing \\
\hline $05-09$ & Mining and Quarrying \\
\hline $10-12$ & Food Products, Beverages and Tobacco \\
\hline $13-15$ & Textiles, Wearing Apparel, Leather and Related Products \\
\hline $16-18$ & Wood and Paper Products, and Printing \\
\hline 19 & Coke and Refined Petroleum Products \\
\hline 20 & Chemicals and Chemical Products \\
\hline 21 & Pharmaceutical Products and Pharmaceutical Preparations \\
\hline $22-23$ & Rubber and Plastics Products; Non-Metallic Mineral Products \\
\hline 24-25 & Metals and Metal Products, Except Machinery and Equipment \\
\hline 26 & Computer, Electronic and Optical Products \\
\hline 27 & Electrical Equipment \\
\hline 28 & Machinery and Equipment N.E.C. \\
\hline $29-30$ & Transport Equipment \\
\hline $31-33$ & Furniture; other Manufacturing; Repair and Installation \\
\hline 35 & Electricity, Gas, Steam and Air Conditioning Supply \\
\hline $36-39$ & Water Supply; Sewerage, Waste Management and Remediation Activities \\
\hline $41-43$ & Construction \\
\hline $45-47$ & Wholesale and Retail Trade, Repair of Motor Vehicles and Motorcycles \\
\hline $49-53$ & Transportation and Storage \\
\hline $55-56$ & Accommodation and Food Service Activities \\
\hline $58-60$ & Publishing, Audiovisual and Broadcasting Activities \\
\hline 61 & Telecommunications \\
\hline $62-63$ & It and other Information Services \\
\hline 64-66 & Financial and Insurance Activities \\
\hline 68 & Real Estate Activities \\
\hline $69-71$ & $\begin{array}{l}\text { Legal and Accounting Activities; Activities of Head offices; Management Consultancy } \\
\text { Activities; Architecture and Engineering Activities; Technical Testing and Analysis }\end{array}$ \\
\hline 72 & Scientific Research and Development \\
\hline 73-75 & $\begin{array}{l}\text { Advertising and Market Research; other Professional, Scientific and Technical } \\
\text { Activities; Veterinary Activities }\end{array}$ \\
\hline $77-82$ & Adminis trative and Support Service Activities \\
\hline 84 & Public Administration and Defence; Compulsory Social Security \\
\hline 85 & Education \\
\hline 86 & Human Health Activities \\
\hline $87-88$ & Residential Care and Social Work Activities \\
\hline $90-93$ & Arts, Entertainment and Recreation \\
\hline $94-96$ & Other Service Activities \\
\hline $97-98$ & $\begin{array}{l}\text { Activities of Hous eholds as Employers; Undifferentiated Goods- and Services- } \\
\text { Producing Activities of Households for Own Use }\end{array}$ \\
\hline 99 & Activities of Extraterritorial Organizations and Bodies \\
\hline
\end{tabular}




\section{REFERENCES}

Bloom, N., and J. Van Reenen (2002) "Patents, Real Options and Firm Performance". Economic Journal, 112: C97-C116.

Griliches, Z. (1998), "Patent Statistics as Economic Indicators: A Survey" in Griliches, Z. (Ed), R\&D and Productivity: The Econometric Evidence, University of Chicago Press.

Hall, B. H., Griliches, Z. and J. A. Hausman (1986), "Patents and R\&D: Is There A Lag?". International Economic Review, 27(2): 265-284.

Hall, B. H., and D. Harhoff (2012), "Recent Research on the Economics of Patents". National Bureau of Economic Research Working Paper No. 17773, NBER, Cambridge (MA).

Helmers, C., and M. Rogers (2011), “Does Patenting Help High-Tech Start-Ups?”. Research Policy, 40(7): 1016-1027.

Levenshtein, V. I. (1965), "Binary Codes Capable of Correcting Deletions, Insertions and Reversals". Doklady Akademii. Nauk SSSR 163(4): p. 845-848, 1965

Pakes, A., and M. Schankerman (1984), "The Rate of Obsolescence of Patents, Research Gestation Lags, and the Private Rate of Return to Research Resources" in Griliches Z. (ed), $R \& D$, Patents, and Productivity, University of Chicago Press.

Ragoussis, A., and E. Gonnard (2012), “The OECD-ORBIS Database: Treatment and Benchmarking Procedures". Version January 2012. Mimeo.

Winkler, W. E. (1999). "The State of Record Linkage and Current Research Problems". Statistics of Income Division, Internal Revenue Service Publication R99/04. www.census.gov/srd/papers/pdf/rr99-04.pdf .

Wagner, S., and I. Cockburn (2010), "Patents and the Survival of Internet-related IPOs". Research Policy, 39(2): 214-228. 